\title{
On the Injectivity of Cycle Maps
}

By

\author{
Morihiko SAITO*
}

\section{Introduction}

Let $X$ be a smooth projective variety over $C$, and $D^{b} \operatorname{MHM}(X)^{\text {go }}$ the bounded derived category of mixed Hodge Modules of geometric origin on $X$, cf. [17]. We have naturally $\boldsymbol{Q}_{X}^{I} \in D^{b} \operatorname{MHM}(X)^{\circ 0}$, whose underlying $\boldsymbol{Q}$-complex is $\boldsymbol{Q}_{X}$. Let

$$
H_{\mathrm{MH}}^{\imath}(X, \boldsymbol{Q}(j))=\operatorname{Ext}_{D b \mathrm{MHM}(X)}^{i} \operatorname{go}\left(\boldsymbol{Q}_{X}^{H}, \boldsymbol{Q}_{X}^{H}(j)\right),
$$

where $(j)$ denotes the Tate twist, cf. [loc. cit.]. Let $\mathrm{CH}^{p}(X)_{\boldsymbol{Q}}$ be the Chow group of $X$ with $Q$-coefficients. We have a cycle map

$$
\mathrm{cl}^{\mathrm{MH}}: \mathrm{CH}^{p}(X)_{Q} \longrightarrow H_{\mathrm{MH}}^{2 p}(X, \boldsymbol{Q}(p)) \text {. }
$$

By adjunction for $a_{X}: X \rightarrow \mathrm{pt}$, we have

$$
H_{\mathrm{MH}}^{\imath}(X, \boldsymbol{Q}(j))=\operatorname{Ext}_{D^{b} \mathrm{MHS}(Q)}^{\iota} \operatorname{go}\left(\boldsymbol{Q}^{H}, \boldsymbol{R} \Gamma\left(X, \boldsymbol{Q}_{X}^{H}\right)(j)\right),
$$

where $\boldsymbol{R} \Gamma\left(X, \boldsymbol{Q}_{X}^{H}\right)=\left(a_{X}\right)_{*} \boldsymbol{Q}_{X}^{I I}$ and $\boldsymbol{Q}^{H}=\boldsymbol{Q}_{\mathrm{pt}}^{H}$. Here $\operatorname{MHS}(\boldsymbol{Q})^{\text {go }}$ is the category of mixed Hodge structures of geometric origin, which is defined by $\operatorname{MHM}(\mathrm{pt})^{\mathrm{go}}$. By [17, II, (4.5)], the canonical filtration $\tau$ (cf. [7]) on $\boldsymbol{R} \Gamma\left(X, \boldsymbol{Q}_{X}^{H}\right)$ splits non canonically, and induces a decreasing filtration $L$ on $H_{\mathrm{MH}}^{i}(X, \boldsymbol{Q}(j))$ such that

$$
\operatorname{Gr}_{L}^{k} H_{\mathrm{MH}}^{\imath}(X, \boldsymbol{Q}(j))=\operatorname{Ext}_{\mathrm{MHS}(\boldsymbol{Q})}^{k} \mathrm{go}\left(\boldsymbol{Q}^{H}, H^{i-k}(X, \boldsymbol{Q})(j)\right) .
$$

Let $L$ denote also the induced filtration on $\mathrm{CH}^{p}(X)_{Q}$. In $[17, \mathrm{II}]$ we showed that the cycle map (0.1) is surjective if the Hodge conjecture is true for any smooth projective varieties. In this case, the $\mathrm{Gr}_{L}^{k} \mathrm{cl}^{\mathrm{MH}}$ are bijective, and $(0.2)$ holds with the left hand side replaced by $\operatorname{Gr}_{L}^{k} \mathrm{CH}^{p}(X)_{Q}$, and $i, j$ by $2 p, p$ (but $L$ may be non separated). The existence of such a filtration was suggested by Bloch [4]. The injectivity of $(0.1)$ is equivalent to the separatedness of the filtration $L$ on $\mathrm{CH}^{p}(X)_{\boldsymbol{Q}}$, and would imply Bloch's conjecture [4]. The bijectivity of $(0.1)$ is related with a problem that $\mathrm{MM}(\operatorname{Spec} \boldsymbol{C}, \boldsymbol{Q})$, the category of (still conjectural) mixed motives (cf. [1]) with base field $\boldsymbol{C}$ and $\boldsymbol{Q}$-coefficients, might be close to the category of mixed Hodge structures of geometric origin

Received October 24, 1990. Revised April 16, 1991.

1991 Mathematics Subject Classification: 14C30.

* Research Institute for Mathematical Sciences, Kyoto University, Kyoto 606 Japan. 
$\operatorname{MHS}(Q)^{\text {go }}$, cf. also $[9][10][12][13]$, etc. It would be also related with Deligne's remark [13, 4.16] and Murre's results [14], cf. [17, II, (3.4)].

In this paper we study the injectivity of $(0.1)$, and reduce it to the surjectivity of some morphisms. Here the notion of geometric level plays an important role, and we can get a strong control on it if the Hodge conjecture is true, cf. (0.11-12) below. We say that a pure Hodge Module with strict support $Z$ has geometric level $\leqq n$, if it is isomorphic to a direct factor of $H^{i} f_{*}\left(\boldsymbol{Q}_{X}^{H}[\operatorname{dim} X]\right)(m)$ for a projective morphism $f: X \rightarrow Z$ such that $X$ is smooth of dimension $\leqq n$. A mixed Hodge Module has geometric level $\leqq n$, if so are $\operatorname{Gr}_{k}^{I V} M$. Let $X$ be a complex algebraic variety, and $\operatorname{MHM}(X)_{\mathrm{g} I \leq n}^{\mathrm{go}}$ the full subcategory of $\operatorname{MHM}(X)^{\text {go }}$ consisting of mixed Hodge Modules of geometric origin on $X$ with geometric level $\leqq n$. We denote $\operatorname{Ext}_{D^{b} \mathcal{A}}^{i}$ by $\operatorname{Ext}_{X, \mathrm{gl} \leqq n}^{i}$ and $\operatorname{Ext}_{X, \text { go }}^{i}$ if $\mathcal{A}=\operatorname{MHM}(X)_{\mathrm{g} 1 \leq n}^{\mathrm{go}}$ and $\operatorname{MHM}(X)^{\mathrm{go}}$ respectively (same for Hom).

Let $X$ be a complex algebraic variety of dimension $\leqq n$. Then we have $\boldsymbol{Q}_{X}^{H}, \boldsymbol{D}_{X}^{H} \in D^{b} \operatorname{MHM}(X)_{\mathrm{g} 1 \leq n}^{\mathrm{go}}$, cf. (2.8), where $\boldsymbol{D}_{X}^{H}$ is the dual of $\mathbf{Q}_{X}^{H}$. By the same argument as $[17, \mathrm{II}]$, we can construct naturally cycle maps

$$
\begin{aligned}
& \mathrm{cl}^{\mathrm{MH}}: \mathrm{CH}_{d}(X)_{\boldsymbol{Q}} \longrightarrow \operatorname{Ext}_{X, \mathrm{~g} 1 \leqq n}^{-2 d}\left(\boldsymbol{Q}_{X}^{H}, \boldsymbol{D}_{X}^{H}(-d)\right), \\
& \mathrm{cl}^{\mathrm{MH}}: \mathrm{CH}_{d}(X)_{\boldsymbol{Q}} \longrightarrow \operatorname{Ext}_{X, \mathrm{go}^{2}}^{-2 d}\left(\boldsymbol{Q}_{X}^{H}, \boldsymbol{D}_{X}^{H}(-d)\right),
\end{aligned}
$$

cf. (2.15), such that (0.4) is the composition of (0.3) with the natural morphism

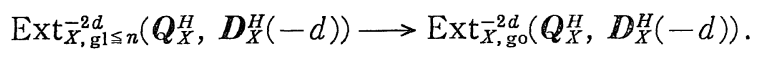

Note that (0.4) coincides with $(0.1)$ by the natural isomorphism $\mathbb{D}_{X}^{H}=\mathbb{Q}_{X}^{H}(\operatorname{dim} X)$ $[2 \operatorname{dim} X]$ if $X$ is smooth, where $p=\operatorname{dim} X-d$. We have

(0.6) Theorem (cf. (3.9-10)). Let $X$ be a pure dimensional quasiprojective variety such that $X$ is locally complete intersection (or, more generally, $Q_{X}[\operatorname{dim} X]$ is a perverse sheaf). Then the cycle map (0.3) for $n=\operatorname{dim} X$ is bijective if $d \geqq$ $\operatorname{dim} X-2$.

So the injectivity of $(0.1)$ is reduced to that of $(0.5)$ if $p \leqq 2$. In general, we can show

(0.7) Theorem (cf. (3.1)). Let $X$ be as above, and $d \in N$. The cycle map (0.3) for $n=\operatorname{dim} X$ is iniective, if (0.5) for $n=\operatorname{dim} Y$ is injective for any closed subvariety $Y$ of $X$ with pure dimension $<\operatorname{dim} X$ such that $Y$ is locally complete intersection (or, more generally, $Q_{Y}[\operatorname{dim} Y]$ is a perverse sheaf) and if the cycle map (0.4) for $d+1$ is surjective for any smooth projective variety with dimension $\leqq \operatorname{dim} X$.

Note that the last hypothesis follows from the Hodge conjecture, cf. [17, II] and (1.4) below. As to the first hypothesis, we have 
(0.8) Proposition (cf. (2.14)). The mor phism (0.5) is injective for an algebraic variety $X$ of dimension $\leqq n$, if the natural morphisms

$$
\begin{gathered}
\operatorname{Ext}_{Y, \operatorname{gl} 1 \leqq n}^{2 p-1}\left(\boldsymbol{Q}_{Y}^{H}, \boldsymbol{Q}_{Y}^{H}(p)\right) \longrightarrow \operatorname{Ext}_{Y, \operatorname{go}}^{2 p-1}\left(\boldsymbol{Q}_{Y}^{I}, \boldsymbol{Q}_{Y}^{H}(p)\right) \\
\operatorname{Ext}_{Y, \operatorname{g} 1 \leqq \operatorname{dim} Y}^{2 p}\left(\boldsymbol{Q}_{Y}^{H}, \boldsymbol{Q}_{Y}^{H}(p)\right) \longrightarrow \operatorname{Ext}_{Y, \mathbf{g} 1 \leqq n}^{2 p}\left(\boldsymbol{Q}_{Y}^{H}, \boldsymbol{Q}_{Y}^{H}(p)\right)
\end{gathered}
$$

are surjective for any smooth (locally closed) subvariety $Y$ of $X$, where $p=\operatorname{dim} I^{\circ}$ $-d$. In particular, (0.5) is injective if $d=n-1$.

Here (0.9) (resp. (0.10)) is always surjective if $p \leqq 1$ (resp. 0). Note that the surjectivity of $(0.10)$ follows from the Hodge conjecture, cf. (0.12) below.

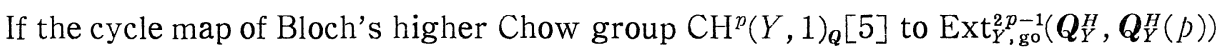
is surjective, it would imply the surjectivity of (0.9), cf. (3.12).

We prove Theorem $(0.7)$ by induction on $\operatorname{dim} X$, and reduce it to the case $X$ smooth and $d=\operatorname{dim} X-1$, cf. $\S 3$. The same argument would apply to the case of reasonable mixed sheaves, cf. (3.13). For the surjectivity of $(0.10)$, we show that the natural morphism

$$
\mathrm{CH}_{\mathrm{dim} Y-p}\left(X \times{ }_{S} Y\right)_{\boldsymbol{Q}} \longrightarrow \operatorname{Ext}_{S, \mathrm{go}}^{2 p}\left(\int_{*} \boldsymbol{Q}_{X}^{H}, g_{*} \boldsymbol{Q}_{Y}^{H}(p)\right)
$$

is surjective if the Hodge conjecture is true, where $f: X \rightarrow S, g: Y \rightarrow S$ are projective morphisms of smooth varieties $X, Y$. Here we need the Hodge conjecture for any smooth projective varieties by definition of mixed Hodge Modules of geometric origin. By induction, the surjectivity of (0.11) implies that of the natural morphisms

$$
\operatorname{Ext}_{X, \mathrm{~g} 1 \leqq n}^{2}(M, N) \longrightarrow \operatorname{Ext}_{X, \mathrm{~g} 1 \leqq m}^{2}(M, N) \longrightarrow \operatorname{Ext}_{X, \mathrm{go}}^{2}(M, N)
$$

for $m>n$, where $M, N$ are pure Hodge Modules of geometric origin with geometric level $\leqq n$ such that the weight of $M$ is equal to the weight of $N$ plus $i$.

In $\S 1$, we introduce the notion of relative correspondence over a base variety, and show the surjectivity of (0.11) assuming the Hodge conjecture. In $\S 2$, we study the property of geometric level. In (2.7) (2.9) we deduce the surjectivity of $(0.12)$ from that of $(0.11)$. We show (0.8) in (2.14). In $\S 3$, we prove $(0.7)$ by induction on $\operatorname{dim} X$, using the Yoneda extension class associated with a cycle.

In this paper, variety means a separated and reduced algebraic variety over $C$ unless otherwise stated.

\section{$\S 1$. Relative Correspondence}

(1.1) Let $f: X \rightarrow S, g: Y \rightarrow S$ be proper morphisms of algebraic varieties, and $d \equiv N$. We define the group of relative correspondences of $X$ to $Y$ over $S$ with $Q$-coefficients by 


$$
C_{S}(X, Y ; \boldsymbol{Q})_{d}=\mathrm{CH}_{d}\left(X \times{ }_{S} Y\right)_{\boldsymbol{Q}} .
$$

By $[17, \mathrm{II}]$ we have a cycle class map

$$
\begin{aligned}
C_{S}(X, Y ; \boldsymbol{Q})_{d} \longrightarrow & \operatorname{Ext}_{X \times{ }^{Y}, \mathrm{go}}^{2 d}\left(\boldsymbol{Q}_{X \times S^{Y}}^{I I}, \boldsymbol{D}_{X \times S^{Y}}^{H}(-d)\right) \\
& =\operatorname{Ext}_{\bar{X} \times Y, \mathrm{go}}^{2 d}\left(\boldsymbol{Q}_{X \times Y}^{H}, \boldsymbol{D}_{X \times S^{Y}}^{H}(-d)\right),
\end{aligned}
$$

where the last isomorphism follows from the adjunction, and is induced by the restriction morphism $\boldsymbol{Q}_{X \times Y}^{H} \rightarrow \boldsymbol{Q}_{X S_{S} Y}^{I}$. (In this paper, the direct image by a closed embedding is sometimes omitted because the equivalence of categories [16, (4.2.10)] holds for mixed Hodge Modules of geometric origin.) We have canonical isomorphisms

$$
\begin{aligned}
& \operatorname{Ext}_{X \times Y, \mathrm{go}}^{-2 d}\left(\boldsymbol{Q}_{X \times Y}^{H}, \boldsymbol{D}_{X \times S_{Y}}^{H}(-d)\right)=\operatorname{Ext}_{S \times S, \mathrm{go}}^{-2 d}\left((f \times g)_{*} \mathbb{Q}_{X \times Y}^{H}, \delta_{*} \mathbb{D}_{S}^{H}(-d)\right) \\
& \quad=\operatorname{Ext}_{S, \mathrm{go}}^{-2 d}\left(f_{*} \boldsymbol{Q}_{X}^{H} \otimes g_{*} \boldsymbol{Q}_{Y}^{H}, \boldsymbol{D}_{S}^{H}(-d)\right)=\operatorname{Ext}_{\bar{S}, \mathrm{go}}^{-2 d}\left(f_{*} \mathbb{Q}_{X}^{H}, g_{*} \boldsymbol{D}_{Y}^{H}(-d)\right),
\end{aligned}
$$

where $\delta: S \rightarrow S \times S$ denotes the diagonal embedding. In fact, the first isomorphism follows from the adjunction for $(f \times g)^{!},(f \times g)_{*}$ together with the isomorphism $\boldsymbol{D}_{X \times{ }_{S} Y}^{H}=(f \times g) \delta_{*} \boldsymbol{D}_{S}^{H}$, the second from the definition of tensor $\otimes$ together with $(f \times g)_{*} \boldsymbol{Q}_{X \times Y}^{H}=f_{*} \boldsymbol{Q}_{X}^{H} \bigotimes g_{*} \mathbf{Q}_{Y}^{H}$, and the last from $[18,2.8]$ using the duality $\boldsymbol{D}\left(g_{*} \boldsymbol{Q} H\right)=g_{*} \boldsymbol{D}_{Y}^{H}$.

Let $Z$ be a reduced and irreducible variety of dimension $d$ with a projective morphism $h: Z \rightarrow S$ factorized by $X \times{ }_{S} Y \rightarrow S$, i. e. $Z$ has morphisms to $X, Y$ over $S$. Then we have a natural morphism

$$
f_{*} \mathbf{Q}_{X}^{H} \longrightarrow h_{*} \boldsymbol{Q}_{Z}^{H} \longrightarrow h_{*} \boldsymbol{D}_{Z}^{H}(-d)[-2 d] \longrightarrow g_{*} \mathbb{D}_{Y}^{H}(-d)[-2 d]
$$

in $D^{b} \mathrm{MHM}(S)^{\mathrm{go}}$, where the first and last morphisms are induced by the restriction and Gysin morphisms [17, II, (2.3.1)], and the middle by $\mathbb{Q}_{Z}^{H} \rightarrow \mathbb{D}_{Z}^{H}(-d)[-2 d]$, cf. (1.1.6) below. Similarly, we have a morphism

$$
\boldsymbol{Q}_{X \times Y}^{H} \longrightarrow \pi_{*} \boldsymbol{Q}_{Z}^{H} \longrightarrow \pi_{*} \boldsymbol{D}_{Z}^{H}(-d)[-2 d] \longrightarrow \mathbb{D}_{X}^{H}{ }_{S^{Y}}(-d)[-2 d],
$$

where $\pi: Z \rightarrow X \times{ }_{S} Y$. Note that, if $Z$ is a subvariety of $X \times{ }_{s} Y$, (1.1.5) coincides with the image of the cycle $[Z]$ by (1.1.2).

Remark. Let $X$ be a variety of dimension $d, X_{i}(1 \leqq i \leqq k)$ the irreducible components of dimension $d$, and $X^{\prime}=\cup_{i} X_{i}$. Then $\mathrm{IC}_{X^{\prime}} \mathbb{Q}^{H}=\bigoplus_{i} \mathrm{IC}_{X_{i}} Q^{H}$ by definition, and we have natural morphisms

$$
\boldsymbol{Q}_{X}^{H}[d] \longrightarrow \mathrm{IC}_{X}, \boldsymbol{Q}^{H} \longrightarrow \mathbb{D}_{X}^{H}(-d)[-d] .
$$

These two morphisms are dual of each other, and induce natural isomorphisms (cf. $[16,(4.5 .14)][17, \mathrm{II},(2.2 .4)])$

(1.1.7) $\operatorname{Hom}_{X, \operatorname{go}}\left(\boldsymbol{Q Q}_{X}^{H}[d], \mathbb{D}_{X}^{H}(-d)[-d]\right) \stackrel{\sim}{\sim} \operatorname{Hom}_{X, \mathrm{ro}}\left(\mathrm{IC}_{X}, \mathbb{Q}^{H}, \boldsymbol{D}_{X}^{H}(-d)[-d]\right)$

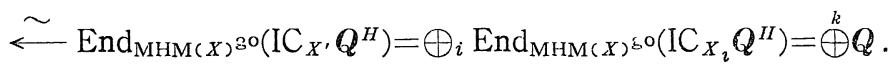


Let $\pi: Y^{\prime} \rightarrow X$ a resolution of singularity. Then the natural morphism $\boldsymbol{Q}_{X}^{I I} \rightarrow$ $D_{X}^{H}(-d)[-2 d]$ of (1.1.6) coincides with the composition of the restriction and Gysin morphisms $\boldsymbol{Q}_{X}^{H} \rightarrow \pi_{*} \boldsymbol{Q}_{Y}^{H}$ and $\pi_{*} \mathbb{Q}_{Y}^{H}=\pi_{*} \boldsymbol{D}_{Y}^{H}(-d)[-2 d] \rightarrow \boldsymbol{D}_{X}^{H}(-d)[-2 d]$ in $D^{b} \operatorname{MHM}(X)^{g \circ}$ by (1.1.7), because it implies that elements of these groups are uniquely determined by their restriction to a generic point of each $X_{\imath}$.

(1.2) Proposition. The morphism (1.1.4) corresponds to (1.1.5) by the isomorphism (1.1.3).

Proof. The last two isomorphisms of (1.1.3) are functorial by [18], and we have a commutative diagram

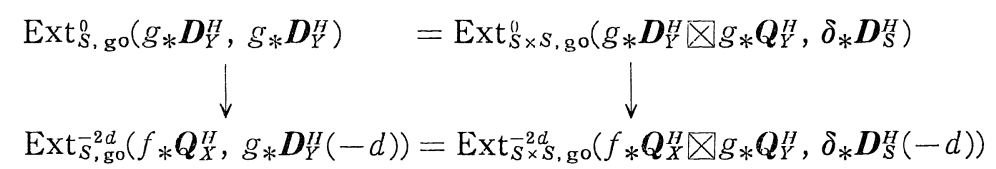

where the vertical morphisms are induced by (1.1.4). We have to show that the image of $\mathrm{id} \in \operatorname{Ext}_{S, \text { go }}^{0}\left(g_{*} \boldsymbol{D}_{Y}^{I I}, g_{*} \boldsymbol{D}_{Y}^{H}\right)$ in the last group corresponds to (1.1.5) by the first isomorphism of (1.1.3).

We can check that the isomorphism $[18,2.8]$ is compatible with direct image, using an acyclic resolution in the definition of direct image, cf. also Remark below. So the morphism $g_{*} \boldsymbol{D}_{Y}^{H} \bigotimes g_{*} \boldsymbol{Q}_{Y}^{H} \rightarrow \boldsymbol{\delta}_{*} \boldsymbol{D}_{S}^{H}$ corresponding to the identity on $g_{*} \boldsymbol{D}_{Y}^{I}$ is the composition

$$
(g \times g)_{*}\left(\boldsymbol{D}_{Y}^{H} \bigotimes \boldsymbol{Q}_{Y}^{H}\right) \longrightarrow \boldsymbol{\delta}_{*} g_{*} \boldsymbol{D}_{Y}^{I I} \longrightarrow \boldsymbol{\delta}_{*} \boldsymbol{D}_{S}^{H},
$$

where the first morphism is induced by $\delta^{*}\left(\boldsymbol{D}_{Y}^{H} \bigotimes \boldsymbol{Q}_{Y}^{H}\right)=\boldsymbol{D}_{Y}^{H}$ with $\delta: Y \rightarrow Y \times Y$ the diagonal embedding. (Note that $\boldsymbol{D}_{Y}^{H} \bigotimes \boldsymbol{Q}_{Y}^{H}=\operatorname{pr}_{1}^{*} \boldsymbol{D}_{Y}^{H}$ by definition, and $\operatorname{pr}_{1} \circ \delta=$ id. We use also $\operatorname{Hom}\left(\boldsymbol{D}_{Y}^{H}, \boldsymbol{D}_{Y}^{H}\right)=\operatorname{Hom}\left(\left(a_{Y}\right)_{1} \boldsymbol{D}_{Y}^{H}, \boldsymbol{Q}^{H}\right)=\boldsymbol{Q}$ if $Y^{Y}$ is connected.) On the other hand, the morphism $(f \times g)_{*} \boldsymbol{Q}_{X^{\prime} Y}^{H} \rightarrow \delta_{*} \boldsymbol{D}_{S}^{H}(-d)[-2 d]$ corresponding to (1.1.5) by the first isomorphism of (1.1.3) is the composition

$$
(f \times g)_{*} \boldsymbol{Q}_{X \times Y}^{I I} \longrightarrow \delta_{*} h_{*} \boldsymbol{Q}_{Z}^{I I} \longrightarrow \delta_{*} h_{*} \boldsymbol{D}_{Z}^{H}(-d)[-2 d] \longrightarrow \delta_{*} \boldsymbol{D}_{S}^{H}(-d)[-2 d] .
$$

The first and last morphisms are naturally factorized by using the morphisms

$$
(f \times g)_{*} \boldsymbol{Q}_{X>Y}^{H} \longrightarrow(h \times g)_{*} \boldsymbol{Q}_{Z \wedge Y}^{H} \longrightarrow \delta_{*} h_{*} \boldsymbol{Q}_{Z}^{I I} \quad \text { and } \quad h_{*} \boldsymbol{D}_{Z}^{H} \longrightarrow g_{*} \boldsymbol{D}_{Y}^{I} \longrightarrow \boldsymbol{D}_{S}^{H},
$$

where the first two morphisms are induced by the restriction morphisms by $Z \rightarrow Z \times Y \rightarrow X \times Y$. Since we have a commutative diagram

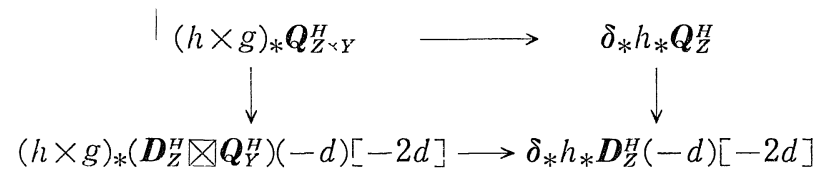

the assertion is reduced to the following commutative diagram 


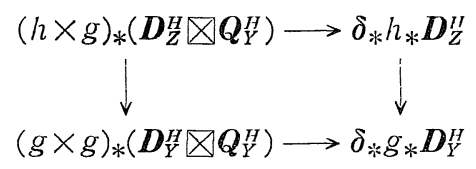

where the horizontal and vertical morphisms are induced by the restriction and Gysin morphisms respectively. But this can be checked easily, because $Z$ is the fiber product of $Z \times Y$ and $Y$ over $Y \times Y$.

Remark. The isomorphism [18, (2.8.1)] is valid also for mixed Hodge Modules of geometric origin. For a morphism of complexes $M \rightarrow N$, the corresponding element of $\operatorname{Hom}\left(M \bigotimes \boldsymbol{D} . \mathrm{V}, \boldsymbol{D}_{\rfloor}^{H}\right)$ is defined by using the edge morphism of the spectral sequence converging to $\operatorname{Hom}\left(L, D_{J}^{I I}\right)$, whose $E_{1}$-term is $\operatorname{Ext}^{q}\left(L^{-p}\right.$, $\left.\boldsymbol{D}_{j}^{H}\right)$, where $L=M \nabla \boldsymbol{D} N$ and $\Delta$ denotes the diagonal. In fact, a morphism $M \rightarrow$ $N$ defines a morphism $L^{0} \rightarrow \boldsymbol{D}_{\Delta}^{H}$ whose composition with $L^{-1} \rightarrow L^{0}$ is zero, and this induces the corresponding morphism $L \rightarrow \boldsymbol{D}_{\Delta}^{I J}$.

(1.3) Theorem. Let $\lambda$ be the composition of (1.1.2) and (1.1.3). Then $\lambda$ is surjective if the Hodge conjecture is true for any smooth projective variety.

Proof. This follows from the next proposition and [17, II, (4.7)].

Remark. The Hodge conjecture for a smooth proper variety is easily reduced to the $X$ smooth projective case using Chow's lemma and the compatibility of the (classical) cycle map with direct image.

(1.4) Proposition. Let $X$ be an algebraic variety, and $\pi: X^{\prime} \rightarrow X$ a surjective proper morphism of a smooth variety. Then the cycle map (0.4) is surjective, if it is surjective for a smooth compactification of $X^{\prime}$.

Proof. Put $M=C\left(\pi_{*} \boldsymbol{D}_{X^{\prime}}^{H} \rightarrow \boldsymbol{D}_{X}^{H}\right)$. Then

$$
M \text { has weight }>0 \text {, i.e. } \operatorname{Gr}_{2}^{I V} H^{J} M=0 \text { for } i \leqq j .
$$

In fact, it is enough to show that $i_{x}^{!} M$ has weights $>0$ for any $x \in X$ by $[16,4.6]$, where $i_{x}:\{x\} \rightarrow X$. Let $Y=\pi^{-1}(x)$. Then $i_{x}^{\prime} M=C\left(\left(a_{Y}\right)_{*} D_{Y}^{H} \rightarrow Q^{I I}\right)$ by base change $[16,(4.4 .3)]$, and the assertion follows from the long exact sequence

$$
\rightarrow H^{\imath}\left(a_{Y}\right)_{*} \boldsymbol{D}_{Y}^{H} \longrightarrow H^{\imath} \boldsymbol{Q}^{H} \longrightarrow H^{i} i_{c}^{\prime} M \longrightarrow H^{\imath+1}\left(a_{Y}\right)_{*} \boldsymbol{D}_{Y}^{I I} \longrightarrow H^{\imath+1} \mathbf{Q}^{H I} \rightarrow,
$$

because $\left(a_{Y}\right)_{*} \boldsymbol{D}_{Y}^{H}$ has weights $\geqq 0$ and $H^{0}\left(a_{Y}\right)_{*} \boldsymbol{D}_{Y}^{\prime \prime} \rightarrow H^{0} \boldsymbol{Q}^{H}=\boldsymbol{Q}^{H}$ is surjective.

By (1.4.1) and [17, II, (4.5.1)], we get the surjectivity of

$$
\begin{aligned}
\operatorname{Ext}_{X^{\prime},{ }_{\mathrm{g}}}^{-2 d}\left(\boldsymbol{Q}_{X^{\prime}}^{I I}, \boldsymbol{D}_{X^{\prime}}^{H}(-d)\right) & =\operatorname{Ext}_{X, \mathrm{go}}^{-2 d}\left(\boldsymbol{Q}_{X}^{I I}, \pi_{*} \mathbb{D}_{X^{\prime}}^{H}(-d)\right) \\
& \longrightarrow \operatorname{Ext}_{X,{ }_{\mathrm{g}} \mathrm{g}}^{-2 d}\left(\boldsymbol{Q}_{X}^{H}, \mathbb{D}_{X}^{H}(-d)\right)
\end{aligned}
$$


using the long exact sequence associated with the triangle $\rightarrow \pi_{*} \boldsymbol{D}_{X^{\prime}}^{H} \rightarrow \boldsymbol{D}_{X}^{H} \rightarrow M \rightarrow$. Since the morphism (1.4.3) is compatible with the push-down of cycles by [17, II, (2.4)], the assertion is reduced to the $X$ smooth case.

Let $X^{\prime \prime}$ be a smooth compactification of $X$ with $j: X \rightarrow X^{\prime \prime}$. We have to check the surjectivity of the restriction morphism

$$
\begin{aligned}
\operatorname{Ext}_{X^{\prime}, g_{0}}^{-2 d}\left(\boldsymbol{Q}_{X^{\prime}}^{H}, \boldsymbol{D}_{X^{\prime \prime}}^{I I}(-d)\right) & \longrightarrow \operatorname{Ext}_{X^{2,}, \mathrm{go}}^{-2 d}\left(\boldsymbol{Q}_{X^{\prime}}^{I}, j_{*} \boldsymbol{D}_{X}^{I I}(-d)\right) \\
& =\operatorname{Ext}_{X^{2}, \mathrm{go}^{2 d}}\left(\boldsymbol{Q}_{X}^{I I}, \boldsymbol{D}_{X}^{H}(-d)\right) .
\end{aligned}
$$

By the same argument as above, it is enough to show that $M^{\prime}=C\left(\boldsymbol{D}_{X^{\prime \prime}}^{H} \rightarrow j_{*} \boldsymbol{D}_{X}^{\mu}\right)$ has weights $>0$. But $i_{x}^{\prime} C\left(\boldsymbol{D}_{X^{\prime \prime}}^{I} \rightarrow j_{*} \boldsymbol{D}_{Y}^{H}\right)=i_{x}^{!} \boldsymbol{D}_{Y^{\prime}}^{H}[1]=\boldsymbol{Q}^{H}[1]$ for $x \notin X$ (because $\left.i_{x}^{\prime} j_{*} \boldsymbol{D}_{X}^{H}=0\right)$ and 0 otherwise. So the assertion follows.

\section{$\S 2$. Level of Mixed Hodge Modules}

(2.1) We say that a pure Hodge structure $M$ has level $\leqq n$ if $\max \{p$ : $\left.\operatorname{Gr}_{F}^{p} M \neq 0\right\}-\min \left\{p: \operatorname{Gr}_{F}^{p} M=0\right\} \leqq n$. We say that a pure Hodge structure of geometric origin $M$ has geometric level $\leqq n$ if there exists a smooth projective variety $X$ of dimension $\leqq n$ such that $M$ is a direct factor of $H^{2}\left(X, \boldsymbol{Q}^{I I}\right)(m)$. Grothendieck's generalized Hodge conjecture [11] is equivalent to

(2.1.1) a pure Hodge structure of geometric origin $M$ has level $\leqq n$ iff it has geometric level $\leqq n$

modulo the Hodge conjecture. In fact, if a pure Hodge structure of geometric origin $M$ has level $\leqq n, M$ is a direct factor of $H^{2}\left(X, Q^{H}\right)(m)$ with $i \leqq n$ assuming Grothendieck's generalized Hodge conjecture, and then we can use the weak Lefschetz theorem to decrease $\operatorname{dim} X$.

We say that a mixed Hodge structure has (geometric) level $\leqq n$ if so are its graded pieces. We say that a mixed Hodge structure has (geometric) level $n$, if it has (geometric) level $\leqq n$ and not $\leqq n-1$. Let $\operatorname{MHS}(\boldsymbol{Q})_{\leqq n}$ (resp. MHS

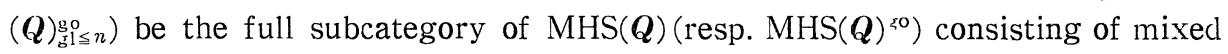
Hodge structures with level (resp. geometric level) $\leqq n$. Put $\operatorname{MHS}(\boldsymbol{Q}) \stackrel{\text { go }}{\underline{\text { o }}}=\mathrm{MHS}$ $(\boldsymbol{Q})^{\text {go }} \cap \operatorname{MHS}(\boldsymbol{Q})_{\cong n}$.

Similarly we can define the notion of (geometric) level $\leqq n$ (and level $n$ ) for mixed Hodge Modules on an algebraic variety $X$. We say that a pure Hodge Module with strict support $Z$ has level $\leqq n$ if its generic variation of Hodge structure has level $\leqq n-\operatorname{dim} Z$, and geometric level $\leqq n$ if it is a direct factor of $H^{i} f_{*} Q_{Y}^{I I}(m)$ for a projective morphism $f: Y \rightarrow Z$ with $Y$ a smooth

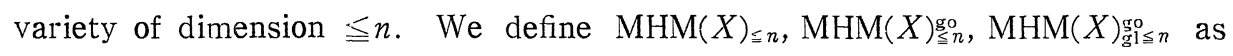
above. These categories are stable by the dual functor $\boldsymbol{D}$, and

(2.1.2) a mixed Hodge Module $M$ has level $\sum n$, if $\varsigma_{s} M$ has level $<n$ for any locally defined function $g$. 
In fact, we may assume $M$ pure by the exactness of $\phi_{\mathrm{g}}$, and $M$ is a variation of Hodge structure. Then the assertion is clear. We have

(2.1.3) the (geometric) level of a pure Hodge Module coincides with its weight $\bmod 2$.

This is clear for level, and follows from Remark (iii) below for geometric level. Generalizing (2.1.1), we might conjecture the following:

(2.1.4) a pure Hodge Module of geometric origin $M$ has level $\leqq n$ iff it has geometric level $\leqq n$.

(2.2) Remarks. (i ) Any pure Hodge Module of geometric origin $M$ with strict support $Z$ is a direct factor of $H^{\jmath} f_{*} Q_{Y}^{I I}(m)$ for a projective morphism $f$ : $Y \rightarrow Z$ such that $Y$ is smooth. In fact, it is enough to show the assertion for the restriction of $M$ to any nonempty open subvariety of $Z$ by Nagata-Hironaka, cf. Remark (ii) below. By [17, I, (2.7)], $M$ is a direct factor of $H^{\jmath} f_{*} Q_{Y}^{H}(m)$ for a projective morphism $f$ of a smooth variety $Y$ to $Z^{\prime}$, where $Z^{\prime}$ is an affine variety containing $Z$ as a closed subvariety (by shrinking $Z$ if necessary). Let $g_{1}, \cdots, g_{k}$ be defining equations of $Z$ in $Z^{\prime}$. Then we may assume that $\cup_{i} f^{-1} g_{i}^{-1}(0)$ is a divisor with normal crossings by replacing $Y$ with its resolution (using the decomposition (2.2.1) below). Then we get the assertion using inductively the commutativity of $\varphi_{g_{i}, 1}$ with $H^{J} f_{*}$ (cf. [16, 2.14]), because $\operatorname{Gr}_{k}^{V} \varphi_{g_{i}, 1} Q_{Y}^{H}\left[d_{Y}\right]$ is a direct sum of constant sheaves supported on intersections of the irreducible components of $g_{\imath}^{-1}(0)$, cf. the proof of [17, II, (4.2)], and the weight filtration on $\varphi_{g_{\ell}, 1} Q_{Y}^{H}\left[d_{Y}\right]$ induces the weight filtration on the direct image. In particular, the geometric level of a mixed Hodge Module of geometric origin is well-defined.

(ii) A pure Hodge Module $M$ with strict support $Z$ has geometric level $\leqq n$, if so is its restriction to a nonempty open subset of $Z$. In fact, the morphism over the open subset can be extended over $Z$ by Nagata-Hironaka, and a pure Hodge Module with strict support is uniquely determined by its restriction to any nonempty open subset of its support.

(iii) If a simple pure Hodge Module $M$ has geometric level $n, M$ is a direct factor of $H^{0} f_{*}\left(Q_{Y}^{H}\left[d_{Y}\right]\right)(m)$ for $f: Y \rightarrow Z$ as above such that $d_{Y}:=\operatorname{dim} Y=n$. In fact, $M$ is a direct factor of $H^{\jmath} f_{*}\left(\boldsymbol{Q}_{Y}^{H}\left[d_{Y}\right]\right)(m)$ with $d_{Y}=n$ and $j \leqq 0$ by the relative hard Lefschetz theorem [15]. If $j<0$, we can decrease $d_{Y}$ by restricting $Y$ to a relative hyperplane $Y^{\prime}$, and $Z$ to a sufficiently small smooth open subset, so that $f$ and the restriction of $f$ to $Y^{\prime}$ are smooth.

(iv) Let $f: X \rightarrow Y$ be a proper morphism, and $M$ a pure Hodge Module on $X$. We have the decomposition

$$
f_{*} M \cong \oplus_{j}\left(H^{\jmath} f_{*} M\right)[-j] \text { in } D^{b} \operatorname{MHM}(Y)
$$

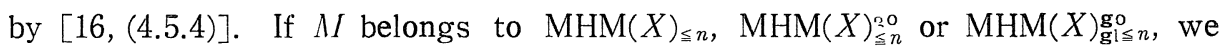




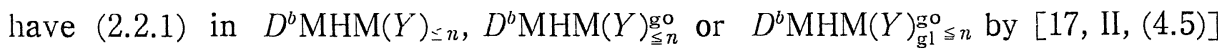
and (2.3) below.

(v) Let $i: X \rightarrow Y$ be a closed embedding of algebraic varieties, and $U=$ $X \backslash Y^{*}$ with the natural inclusion $j: U \rightarrow Y$. Then we have naturally the functors

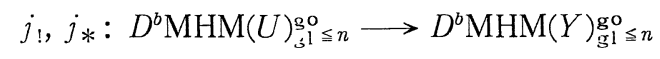

using (2.4.1) below and a Čech covering by affine open subvarieties. They are the left and right adjoint functors of the pull-back $j^{*}=j !$. Similarly we have

$$
i_{* k} i^{*}, i_{*} i^{1}: D^{b} \operatorname{MHM}(X)_{\mathrm{g} 1 \leqq n}^{\mathrm{go}} \longrightarrow D^{b} \operatorname{MHM}(X)_{\mathrm{g} 1 \leqq n}^{\mathrm{go}}
$$

using a Čech covering. Combined with (2.6) below, we get

$$
i^{*}, i^{!}: D^{b} \mathrm{MHM}\left(Y^{\prime}\right)_{S_{1}^{1} \leqq n}^{\mathrm{oo}} \longrightarrow D^{b} \operatorname{MHM}(X)_{s_{1} \leqq n}^{\mathrm{go}} .
$$

They are the left and right adjoint functors of the direct image $i_{*}$ by the same argument as [16, (4.4.1)]. It is the same for $D^{b} \operatorname{MHM}(X)_{\S}^{\mathrm{go}}, D^{b} \operatorname{MHM}(X)_{\leqq n}$, and these functors are compatible with the natural functors $D^{b} \operatorname{MHM}(X)_{\mathrm{g} I \leq n}^{\mathrm{go}} \rightarrow$ $D^{b} \operatorname{MHM}(X) \stackrel{\text { go }}{\subseteq} \rightarrow D^{b} \operatorname{MHM}(X)_{\leqq n} \rightarrow D^{b} \operatorname{MHM}(X)$.

(2.3) Proposition. The derived categories $D^{b} \mathrm{MHM}(X)_{\S n}, D^{b} \mathrm{MHM}(X)_{\unlhd n}^{\mathrm{go}}$, $D^{b} \mathrm{MHM}(X)_{\mathrm{g} 1 \leq n}^{\mathrm{go}}$ are stable by direct images, i.e. for $f: X \rightarrow Y$, we have natural functors $f_{*}, f_{!}: D^{b} \mathrm{MHM}(X)_{2 n} \rightarrow D^{b} \mathrm{MHM}\left(Y^{r}\right)_{\leq n}$, etc. compatible with the natural functors $D^{b} \mathrm{MHM}(X)_{\mathrm{g} 1 \leqq n}^{\mathrm{go}} \rightarrow D^{b} \operatorname{MHM}(X)_{\underline{n}}^{\mathrm{go}} \rightarrow D^{b} \mathrm{MHM}(X)_{\leq n} \rightarrow D^{b} \operatorname{MHM}(X)$.

Proof. By definition of direct image [16] using a sheaf theoretic resolution of Beilinson [2], it is enough to show that $\operatorname{MHM}(X)_{\mathrm{g} 1 \leq n}^{\mathrm{go}}$, etc. are stable by the cohomological direct images $H^{\jmath} f_{:}, H^{\jmath} f_{*}$ for a quasiprojective morphism $f$, because we have (2.2.3). So the assertion follows from the next proposition.

(2.4) Proposition. Let $f: X \rightarrow I^{r}$ be a morphism of algebraic varieties, and II a mixed Hodge Module of level $\leqq n$ on $X$. Then

$$
H^{\jmath} \int_{*} M, H^{\jmath} f_{1} M \text { have level } \leqq n .
$$

Moreover, if $f$ is proper and $M$ is pure with strict support $X$, let $H^{j} f_{*} M=$ $\oplus_{Z} M_{Z}^{J}$ be the decomposition by strict support, cf. [15]. Then

(2.4.2) $M_{Z}^{\prime}$ has level $\leqq n-|i|$ for $Z=f(X)$ and level $<n-|j|$ otherwise.

The same assertions hold with level replaced by geometric level if.$M$ is of geometric origin.

Proof. We may assume $f$ quasiprojective by the same argument as above, and $M$ pure with strict support $X$, using the spectral sequence associated with the weight filtration.

We first show the assertion on geometric level. If $f$ is projective, (2.4.1) is 
clear by definition. If $f$ is an open embedding, it follows from Nagata-Hironaka. For (2.4.2), we may assume $f$ projective using Chow's lemma and (2.2.1). In fact, take a birational projective morphism $\pi: X^{\prime} \rightarrow X$ such that $X$ is quasiprojective. Assume $\pi$ induces an isomorphism over $U \subset X$. Then there exists uniquely a pure Hodge Module $M^{\prime}$ with strict support $X^{\prime}$ such that $\left.L^{\prime}\right|_{\pi^{-1}(U)} \cong$ $\left.M\right|_{U}$ (cf. [16]) and the assertion is reduced to $M^{\prime}$ and $f \pi$. So we may assume $f$ projective. Then the case $Z=f(X)$ is clear by definition using the relative hard and weak Lefschetz theorems, cf. (2.2, iii). If $Z \neq \int(X)$, we take (locally) a nonzero function $g$ on $f(X)$ such that $g^{-1}(0) \supset Z$. Then the assertion follows from the next proposition and the commutativity of $\varphi$ with the direct image (cf. $[16,2.14]$ ), because $\varphi_{g} M_{Z}^{j}=M_{Z}^{j}$ and we have a surjection can : $\psi_{g f} M \rightarrow \varphi_{g f} M$ (cf. $[15,5.1 .4]$ ).

We now show the assertion on level. If $f$ is an open embedding such that $Y \backslash X$ is a locally principal divisor, this follows from the next proposition, using $[16,(2.11 .10)]$. So we may assume $f$ projective, and the assertion is reduced to (2.4.2). By the same argument as above, it is enough to show the case $Z=f(X)$. So we may assume $f$ smooth. By $[16,2.14]$, the assertion is reduced to the case $Y=p t$ by restricting to a generic fiber using the iteration of nearby cycle functors $\phi$. Let $D$ be the singular locus of $M$, i.e. $D$ is the largest subvariety such that the restriction of $M$ to its complement is a variation of Hodge structure. Let $\pi: X^{\prime} \rightarrow X$ be a resolution of $(X, D)$, and $M^{\prime}$ be a pure Hodge structure with strict support $X^{\prime}$ such that $\left.M^{\prime}\right|_{X^{\prime} \backslash D^{\prime}}=\left.M\right|_{X \backslash D}$ where $D^{\prime}=\pi^{-1}(D)$, cf. $[16,3.21]$. By the same argument as above, it is enough to show the assertion for $M^{\prime}$, and we may assume $X$ smooth and $D$ a divisor with normal crossings. Then the assertion follows from the calculation of $[16,3.11]$ (using the intersection of $\mathrm{DR}\left(j_{! *}^{\mathrm{reg}} M, F\right)$ with $K_{*} L$ which is filtered quasi-isomorphic to $\operatorname{DR}(j ! *$ reg $M, F))$.

(2.5) Proposition. Let $g$ be a function. If a mixed Hodge Module $M$ has geometric level $\leqq n$, then $\psi_{g} M$ has geometric level $<n$ and $\varphi_{g} W$ has geometric level $\leqq n$. The same assertion holds with geometric level replaced by level.

Proof. We may assume that $M$ is a pure Hodge Module with strict support because $\dot{\psi}_{g}, \varphi_{g}$ are exact functors. We first show the assertion on geometric level. By $(2.2, \mathrm{i}), M$ is a direct factor of $H^{J} f_{*} Q_{Y}^{H}(m)$ for a projective morphism $f$ of a smooth variety $Y$, and we may assume $f^{-1} g^{-1}(0)$ is a divisor with normal crossings by the same argument as in $(2.2, \mathrm{i})$. Taking a ramified covering, and blowing up further, we may assume the multiplicities of its irreducible components are constant by the semi-stable reduction theorem. Then $\operatorname{Gr}_{i}^{T} \psi_{g} Q_{Y}^{H}$, $\operatorname{Gr}_{l}^{W V} \varphi_{g} Q_{Y}^{I I}$ are direct sums of constant sheaves supported on intersections of the irreducible components of $f^{-1} g^{-1}(0)$. So the assertion follows.

For the assertion on level, let $D$ be the singular locus of $M$, cf. the proof 
of (2.4.1). By the decomposition theorem and the compatibility of direct image with nearby and vanishing cycle functors [16], the assertion is reduced to the case the support is smooth and $D \cup g^{-1}(0)$ is a divisor with normal crossings. The assertion is trivial, if the support is contained in $g^{-1}(0)$. In the other case, the assertion follows from the calculation of nearby cycle functor [16, (3.17.3)] and the surjectivity of can : $\phi_{g} M \rightarrow \varphi_{g} M$.

(2.6) Proposition. Let $X$ be a closed subvariety of $Y$. Then the natural functor

$$
i_{*}: D^{b} \operatorname{MHM}(X)_{\mathrm{g} 1 \leq n}^{\mathrm{go}} \longrightarrow D^{b} \mathrm{MHM}(Y)_{\mathrm{g} 1 \leq n}^{\mathrm{go}}
$$

is fully faithful and its essential image is the full subcategory consisting of the objects $M$ such that $\operatorname{supp} H^{j} M \subset X$ (same for $\left.D^{b} \operatorname{MHM}(X)_{s n}^{g o}, D^{b} \operatorname{MHM}(X)_{s n}\right)$.

Proof. This follows from the same argument as $[16,(4.2 .10)]$ using (2.3) and (2.5).

(2.7) Theorem. Let $M, N$ be pure Hodge Modules of geometric origin with geometric level $\leqq n$ on an algebraic variety $S$. Let $k=\mathrm{wt} M-\mathrm{wt} N$, where $\mathrm{wt} M$ is the weight of $M$. If Hodge coniecture is true for any smooth projective varieties, the composition

$$
\operatorname{Ext}_{S, \mathrm{~g} 1 \leqq n}^{k}(M, N) \longrightarrow \operatorname{Ext}_{S, \mathrm{go}, \leqq n}^{k}(M, N) \longrightarrow \operatorname{Ext}_{S, \mathrm{go}}^{k}(M, N)
$$

is surjective, where $\operatorname{Ext}_{S, \mathrm{go}, \leqq n}^{k}$ denotes $\operatorname{Ext}_{D b_{\llcorner}}^{k}$ for $\mathcal{A}=\operatorname{MHM}(S) \stackrel{\text { go }}{\leqq}$.

Proof. We may assume $M, N$ simple. We proceed by induction on $n$. By (2.2, iii), $M, N$ are direct factors of $H^{0} f_{*}\left(\boldsymbol{Q}_{X}^{H}\left[d_{X}\right]\right)(m), H^{0} g_{*}\left(\boldsymbol{Q}_{Y}^{H}\left[d_{Y}\right]\right)(r)$ for projective morphisms $f: X \rightarrow S, g: Y \rightarrow S$ such that $d_{X}, d_{Y}$ are the level of $M$, $N$. By Remark $(2.2, \mathrm{iv})$, we may replace $M, N$ by $f_{*}\left(\boldsymbol{Q}_{X}^{I I}\left[d_{X}\right]\right)(m), g_{*}\left(\boldsymbol{Q}_{Y}^{H}\left[d_{Y}\right]\right)$ (r). Let

$$
\xi \in \operatorname{Ext}_{S, g o}^{k}\left(f_{*}\left(\boldsymbol{Q}_{X}^{I I}\left[d_{X}\right]\right)(m), \quad g_{*}\left(\boldsymbol{Q}_{Y}^{H}\left[d_{Y}\right]\right)(r)\right) .
$$

It is the image of a cycle $\zeta$ of dimension $d$ by $\lambda$, cf. (1.3), where $d=\left(d_{X}+d_{Y}\right.$ $-k) / 2$. Since the assertion is trivial for $k \leqq 0$, we may assume $k>0$. Then $d<\max \left\{d_{X}, d_{Y}\right\}$. Decomposing $\xi$ into a sum of morphisms, we may assume that $\zeta$ is represented by an irreducible variety $Z$. Replacing $Z$ by its resolution, $\xi$ is obtained by the composition of the restriction and Gysin morphisms, cf. (1.1.4). So the assertion follows from the next proposition.

(2.8) Proposition. Let $X$ be an algebraic variety $X$ of dimension $\leqq n$. Then $\boldsymbol{Q}_{X}^{H}\left(\right.$ resp. $\left.\boldsymbol{D}_{X}^{H}\right)$ is naturally lifted to $D^{b} \mathrm{MHM}(X)_{\mathrm{gl}}^{\mathrm{go}} \leq n$. More precisely, there exists uniquely an object of $D^{b} \operatorname{MHM}(X)_{\mathrm{g} 1 \leq n}^{\mathrm{go}}$, denoted also by $\boldsymbol{Q}_{X}^{H}$ (resp. $\left.\boldsymbol{D}_{X}^{H}\right)$, whose underlying $\boldsymbol{Q}$-complex is $\boldsymbol{Q}_{X}$ (resp. $\boldsymbol{D}_{X}$ ) and whose restruction to a smooth open 
dense subvariety $U$ is isomorphic to $\mathbf{Q}_{U}^{I I}$ (resp. $\boldsymbol{D}_{U}^{H}$ ) in $D^{b} \mathrm{MHM}(U)$. Moreover, for $f: X \rightarrow Y$ a morphism of algebraic varieties of dimension $\leqq n$, the restriction and Gysin morphisms $\boldsymbol{Q}_{Y} \rightarrow f_{*} \boldsymbol{Q}_{X}$ and $f: \boldsymbol{D}_{X} \rightarrow \boldsymbol{D}_{Y}$ are uniquely lifted to morphisms of $D^{b} \mathrm{MHM}\left(Y^{r}\right)_{\mathrm{g} 1 \leqq n}^{\mathrm{go}}$ (and hence $D^{b} \mathrm{MHM}\left(Y^{r}\right)_{\leq n}^{\mathrm{go}}, D^{b} \mathrm{MHM}\left(Y^{r}\right)_{\leqq n}$ ), and they are compatible with the composition of morphisms.

Proof. We show the assertion for $\mathbb{Q}_{X}^{H}$, because the dual argument holds for $\boldsymbol{D}_{X}^{H}$. We may assume $X, Y$ connected. The first assertion is clear if $X$ smooth. If $\boldsymbol{Q}_{X}^{H}$ exists, the natural morphism

$$
\operatorname{Hom}_{X, \mathrm{~g} 1 \leqq n}\left(\boldsymbol{Q}_{X}^{H}, \boldsymbol{Q}_{X}^{H}\right) \longrightarrow \operatorname{Hom}_{\boldsymbol{Q}}\left(\boldsymbol{Q}_{X}, \boldsymbol{Q}_{X}\right)=\boldsymbol{Q}
$$

is injective by Remark below, because $Q_{X}^{H} \in \mathcal{C}$ is clear. Here $C$ and ${ }^{c} H^{k}$ are as in Remark below with $A=\operatorname{MHM}(X)_{\text {gisn }}^{\text {go }}$. For surjectivity, we have a natural injection $\boldsymbol{Q}_{X}^{H} \rightarrow{ }^{c} H^{0} j_{*} \mathbf{Q}_{U}^{H}$ in $\mathcal{C}$ with $j: U \rightarrow X$, and the assertion is reduced to that for $U$ by the argument on the surjectivity of (2.8.3), because $j_{*}$ is left exact with respect to the classical $t$-structure (i. e., $\left.{ }^{c} H^{k} j_{*} \mathbb{Q}_{U}^{I I}=0(k<0)\right)$, and $\operatorname{Hom}\left(\mathbb{Q}_{X}^{H}\right.$, $\left.{ }^{c} H^{0} j_{*} \boldsymbol{Q}_{U}^{H}\right)=\operatorname{Hom}\left(\boldsymbol{Q}_{X}^{H}, j_{*} \boldsymbol{Q}_{U}^{H}\right)=\operatorname{Hom}\left(\boldsymbol{Q}_{U}^{H}, \boldsymbol{Q}_{U}^{H}\right)$. This argument shows also uniqueness of $Q_{X}^{I I}$. For existence, we may assume the existence on an open subvariety $X^{\prime}$ of $X$ such that $Z:=X \backslash X^{\prime}$ is smooth and $X$ is topologically locally trivial along $Z$ by inductive argument using a Whitney stratification of $X$. Let $i_{*} M=C\left(Q_{X}^{H} \rightarrow^{c} H^{0} j_{*}^{\prime} Q_{X^{\prime}}^{H}\right)$ in $D^{b} \operatorname{MHM}(X)$ with $i: Z \rightarrow X$ and $j^{\prime}: X^{\prime} \rightarrow X$. Then $M$ is a variation of Hodge structure of type $(0,0)$ on $Z$, and has geometric level $\operatorname{dim} Z$. So it is enough to show that ${ }^{c} H^{0} j_{*}^{\prime} \mathbf{Q}_{X^{\prime}}^{H} \rightarrow i_{*} M$ is lifted to a morphism of C. By adjunction, it is equivalent to a morphism $i^{*}\left({ }^{c} H^{0} j_{*}^{\prime} Q_{X^{\prime}}^{I I}\right) \rightarrow M$. So the assertion is clear, because $i^{*}$ is exact with respect to the classical $t$-structure so that $i^{*}\left({ }^{c} H^{0} j_{*}^{\prime} Q_{X^{\prime}}^{H}\right) \in \mathcal{C}$, and it is a variation of mixed Hodge structure.

For the restriction morphism, it is enough to show bijectivity of

$$
\operatorname{Hom}_{Y, \mathrm{~g} 1 \leqq n}\left(\boldsymbol{Q}_{Y}^{H}, f_{*} \boldsymbol{Q}_{X}^{H}\right) \longrightarrow \operatorname{Hom}_{D\left(\boldsymbol{Q}_{Y}\right)}\left(\boldsymbol{Q}_{Y}, f_{*} \boldsymbol{Q}_{X}\right) .
$$

Since $\mathscr{H}^{k} f_{*} \boldsymbol{Q}_{X}=0$ for $k<0$, we may replace $f_{*} \boldsymbol{Q}_{X}^{H}, f_{*} \boldsymbol{Q}_{X}$ by ${ }^{c} H^{0} f_{*} \boldsymbol{Q}_{X}^{H}, \mathscr{H}^{0} f_{*} \boldsymbol{Q}_{X}$. Then the injectivity follows from (2.8.3). For surjectivity, we may assume $f(X)$ is dense in $Y^{\prime}$ using the adjunction for the inclusion of the closure of $f(X)$ into $Y$. Let $U$ be a smooth open dense subvariety of $Y$ such that the restriction of $\mathscr{T}^{0} f_{*} \boldsymbol{Q}_{X}$ to $U$ is a local system. Then we have a natural inclusion ${ }^{c} H^{0} f_{*} \boldsymbol{Q}_{X}^{H} \rightarrow$ ${ }^{c} H^{0} j_{*} j^{*}\left({ }^{c} H^{0} f_{*} Q_{X}^{H}\right)$ in $\mathcal{C}$ on $Y$ (because the underlying $Q$-complex of its kernel is zero). By the same argument as above using the surjectivity of (2.8.3), the assertion is reduced to that for the restriction of $f$ over $U$, and we may assume $Y$ is smooth and $\mathscr{H}^{0} f_{*} Q_{X}$ is a local system. Then the surjectivity of (2.8.2) is clear, because ${ }^{c} H^{0} f_{*} Q_{X}^{H}$ is a variation of Hodge structure of type $(0,0)$ on $Y$.

Remark. Let $\mathcal{A}=\operatorname{MHM}(X)_{\mathrm{g} I \leq n}^{\mathrm{go}}, \operatorname{MHM}(X) \underset{s n}{\mathrm{go}}, \operatorname{MHM}(X)_{\S n}$ or $\operatorname{MHM}(X)^{\text {co }}$. Then we have a 'classical' $t$-structure $\left({ }^{c} \mathscr{D}^{\leqq 0},{ }^{c} \mathscr{D}^{\geq 0}\right)$ on $D^{b} A$ such that $M \in \mathscr{D}^{\leq 0}$ (resp. 
$M \in \mathscr{D}^{\geq 0}$ ) if and only if $\mathscr{T}^{j} \operatorname{rat}(M)=0$ for $j>0$ (resp. $j<0$ ), where $\operatorname{rat}(M)$ is the underlying $\boldsymbol{Q}$-complex of $M$ and $\mathscr{H}^{\jmath}$ is the natural (i.e. classical) cohomology functor. In fact, ${ }^{c} \mathscr{D}^{\leqq 0}\left(\right.$ resp. $\left.{ }^{c} \mathscr{D}^{\geq 0}\right)$ is defined by the condition :

For any closed embedding $i_{S}: S \rightarrow X$ of an irreducible variety $S$, there is a non empty open subvariety $U$ of $S$ such that $\left.\left(H^{k} i * M\right)\right|_{U}=0$ for $k>\operatorname{dim} S$ (resp. $\left.\left(H^{k} i_{S}^{!} M\right)\right|_{U}=0$ for $k<\operatorname{dim} S$ ),

using the theory of gluing $t$-structure in [3]. Since the functors $H^{k} i_{S}^{*}, H^{k} i_{S}$ corresponds to the functors ${ }^{p} \mathscr{H}^{k} i{ }_{S}^{*},{ }^{p} \mathscr{H}^{k} i_{S}^{\vdots}$ on the underlying $\boldsymbol{Q}$-complexes by the forgetful functor rat, and rat: $\operatorname{MHM}(X) \rightarrow \operatorname{Perv}\left(\boldsymbol{Q}_{X}\right)$ is faithful and exact, we may replace $H^{k} i_{S}^{*} M, H^{k} i_{S}^{\vdots} M$ by ${ }^{p} \mathcal{H}^{k} i_{S}^{*} \operatorname{rat}(M),{ }^{p} \mathcal{H}^{k} i_{S}^{!} \operatorname{rat}(M)$, and the above condition depends only on the underlying $\boldsymbol{Q}$-complex. Then we can check the coincidence with the above condition. In fact, it is clear for ${ }^{c} \mathscr{D} \leqq 0$ by the distinguished triangle as in $[16,(4.4 .1)]$, and for ${ }^{c} \mathscr{D}^{\geq 0}$ we use also the left exactness of the functor $j_{*}$ with respect to the classical $t$-structure on $D_{c}^{b}\left(\boldsymbol{Q}_{X}\right)$.

Let ${ }^{c} H^{k}: D^{b} \leadsto \rightarrow C$ denote the cohomology functor associated with the classical $t$-structure where $C$ is the heart of the $t$-structure, cf. [3]. Then $M \in D^{b} A$ belongs to $C$ if and only if ${ }^{c} H^{k}(M)=0$ for $k \neq 0$. The functor ${ }^{c} H^{k}$ corresponds to the natural cohomology functor $\mathscr{H}^{k}: D_{c}^{b}\left(\boldsymbol{Q}_{X}\right) \rightarrow M\left(\boldsymbol{Q}_{X}\right)$ by the forgetful functor rat, and the forgetful functor rat: $\mathcal{C} \rightarrow M\left(\boldsymbol{Q}_{X}\right)$ is exact. It is also faithful, because $\operatorname{Im}$ commutes with rat, and $M \in C$ is 0 if and only if $\operatorname{rat}(M)$ is zero (this is checked by restricting to an open subvariety of the support of $M$ ). So we get injectivity of

$$
\operatorname{Hom}_{C}(M, N) \longrightarrow \operatorname{Hom}_{Q}(\operatorname{rat}(M), \operatorname{rat}(N))
$$

for $M, N \in C$. Note that (2.8.3) is surjective if there is an injection $N \rightarrow N^{\prime}$ such that (2.8.3) is surjective for $\left(M, N^{\prime}\right)$. This is checked using the morphism of the long exact sequences associated with the short exact sequence $0 \rightarrow N \rightarrow$ $N^{\prime} \rightarrow N^{\prime} / N \rightarrow 0$.

(2.9) Proposition. For $M, N, k$ as in (2.7), the natural morphism

$$
\operatorname{Ext}_{S, \mathrm{~g} 1 \leqq n}^{k}(M, N) \longrightarrow \operatorname{Ext}_{S, \mathrm{~g} 1 \leqq m}^{k}(M, N)
$$

is surjective for $m>n$, if the Hodge conjecture is true for any smooth projective varieties.

Proof. Let $\xi \in \operatorname{Ext}_{S, \mathrm{~g} 1 \leqq m}^{k}(M, N)$. We apply [17, II, (4.5)] to $\operatorname{MHM}(S)_{\mathrm{g} g 1 \leqq m}^{\mathrm{go}}$, and get pure Hodge Modules $M_{\jmath}(0 \leqq j \leqq k)$ with $\xi_{j} \in \operatorname{Ext}_{S, \mathrm{~g} 1 \leqq m}^{1}\left(M_{j}, M_{\jmath-1}\right)(1 \leqq j \leqq k)$ such that $M=M_{k}, N=M_{0}$, wt $M_{j}=\mathrm{wt} N+j$, and $\xi=\Pi_{j} \xi_{j}$. Let $d_{j}$ be the geometric level of $M_{j}$. By the next lemma, we may assume $d_{\jmath} \leqq \max \left\{d_{\jmath_{-1}}, d_{\jmath_{1}}\right\}$ for $0<$ $j<k$ (by replacing $M_{\jmath}, \xi_{3}$ if necessary), if the Hodge conjecture is true. Since $d_{j}-d_{\jmath-1}$ is odd by (2.1.3), there is an integer $j_{i}$ such that 


$$
d_{j}<d_{\jmath_{-1}} \text { for } i \leqq j_{2} \text { and } d_{j}>d_{\jmath-1} \text { otherwise , }
$$

where $j_{i}$ may be 0 or $k$. So the assertion follows.

(2.10) Lemma. Let $M_{j}(j=0,1,2)$ be pure Hodge Modules of geometric origin with weight $w+j$ and geometric level $\leqq n$ on $S$, and $\xi_{,} \in \operatorname{Ext}_{S, g 1 \leqq n}^{1}\left(M_{\jmath}\right.$, $\left.M_{j-1}\right)(j=1,2)$. If the Hodge conjecture is true for any smooth projective varieties, there exist pure Hodge Modules of geometric origin $M_{j}^{\prime}$ with weight $w+j$ and geometric level $d_{j}^{\prime}(j=0,1,2)$ and $\xi_{j}^{\prime} \in \operatorname{Ext}_{S, \mathrm{~g} 1 \leqq n}^{1}\left(M_{j}^{\prime}, M_{j-1}^{\prime}\right)(j=1,2)$ such that $M_{j}=$ $M_{j}^{\prime}(j=0,2), d_{1}^{\prime} \leqq \max \left\{d_{0}^{\prime}, d_{2}^{\prime}\right\}$ and

$$
\xi_{1} \xi_{2}=\xi_{1}^{\prime} \xi_{2}^{\prime} \text { in } \operatorname{Ext}_{S, g 1 \leqq n}^{2}\left(M_{2}, M_{0}\right) .
$$

Proof. The assertion follows from (2.7) if we replace $(2.10 .1)$ by

$$
\xi_{1} \xi_{2}=\xi_{1}^{\prime} \xi_{2}^{\prime} \text { in } \operatorname{Ext}_{S, \text { go }}^{2}\left(M_{2}, M_{0}\right),
$$

where $\operatorname{Ext}_{S, g 1 \leqq n}^{1}\left(M_{\jmath}, M_{\jmath-1}\right)=\operatorname{Ext}_{S, \text { go }}^{1}\left(M_{\jmath}, M_{j-1}\right)$ by definition. We show that (2.10.2) implies (2.10.1). Let $N_{1}=M_{1} \oplus M_{1}^{\prime}$, and $N_{\jmath}=M_{\jmath}$ for $j=0,2$. Define $\zeta_{\jmath} \in$ $\operatorname{Ext}_{S, \mathrm{gl} \leqq n}^{1}\left(N_{j}, N_{j-1}\right)(j=1,2)$ by $\zeta_{1}=\xi_{1}-\xi_{1}^{\prime}, \zeta_{2}=\xi_{2}+\xi_{2}^{\prime}$. Then, it is enough to show $\zeta_{1} \zeta_{2}=0$ in $\operatorname{Ext}_{S, \mathrm{~g} 1 \leqq n}^{2}\left(M_{2}, M_{0}\right)$ assuming $\zeta_{1} \zeta_{2}=0$ in $\operatorname{Ext}_{S, \text { go }}^{2}\left(M_{2}, M_{0}\right)$. Using the long exact sequence associated with the functor $\operatorname{Ext}\left(M_{2}, *\right)$ and the short exact sequence associated with $\zeta_{1}$, the assertion is equivalent to the existence of a mixed Hodge Module of geometric origin $M$ with geometric level $\leqq n$ such that $\mathrm{Gr}_{w+j}^{W} M=N$, and $\zeta$, is identified with the extension class defined by the short exact sequence $0 \rightarrow \mathrm{Gr}_{w+j-1}^{W} M \rightarrow W_{w+j} M / W_{w+j-2} M \rightarrow \mathrm{Gr}_{w+j}^{W} M \rightarrow 0$. But this follows from the assumption except for the condition on the geometric level of $M$. So the assertion is clear by definition of geometric level on mixed Hodge Modules.

(2.11) Remark. Let $M, N, k$ be as in (2.7), and $d, d^{\prime}$ the geometric level of $M, N$. By (2.9.2), $\zeta \in \operatorname{Ext}_{S, \mathrm{~g} 1 \leqslant n}^{k}(M, N)$ is zero for $k>d+d^{\prime}$, and its restriction to a sufficiently small open subvariety of $S$ is zero for $k>d+d^{\prime}-2 \operatorname{dim} S$, if the Hodge conjecture is true for any smooth projective varieties. This follows by the same argument as in (2.9).

(2.12) Lemma. Let $X$ be an $n$-dimensional algebraic variety such that $\boldsymbol{Q}_{X}[n]$ is a perverse sheaf. Then $\boldsymbol{Q}_{X}^{H}[n], \boldsymbol{D}_{X}^{H}[-n]$ are mixed Hodge Modules (i.e $H^{J} \boldsymbol{Q}_{X}^{H}=$ $H^{-j} \boldsymbol{D}_{X}^{H}=0$ for $j \neq n$ ) with geometric level $n$. Let $Y$ be a locally principal divisor of $X$, i.e. locally $Y=g^{-1}(0)_{\text {red }}$ for a non zero divisor $g$ of $\mathcal{O}_{X}$. Then $Q_{Y}[n-1]$ is a perverse sheaf.

Proof. We first show the last assertion. The vanishing of ${ }^{p} \mathscr{H}^{j} \boldsymbol{Q}_{Y}$ for $j>$ $n-1=\operatorname{dim} Y$ follows from the definition of perverse sheaf [3]. We have locally

$$
\boldsymbol{Q}_{Y}=C\left(\operatorname{can}: \phi_{g, 1} \boldsymbol{Q}_{X} \rightarrow \varphi_{g, 1} \boldsymbol{Q}_{X}\right)[-1] .
$$


This implies ${ }^{p} \mathfrak{H}^{3} Q_{Y^{r}}=0$ for $j<n-1$, because $\phi_{g, 1}[-1], \varphi_{g, 1}[-1]$ are exact functors.

For the first assertion, it is enough to show the assertion for $Q_{X}^{H}$ by duality. Since the functor rat: $\operatorname{MHM}(X) \rightarrow \operatorname{Perv}\left(\boldsymbol{Q}_{X}\right)$ is faithful and rato $H^{j}={ }^{p} \mathscr{H}^{j}$ orat, we have $H^{j} \boldsymbol{Q}_{X}^{H}=0$ for $j \neq n$. The assertion on geometric level is clear by (2.8). This follows also from an exact sequence of mixed Hodge Modules

$$
0 \longrightarrow \boldsymbol{Q}_{Y}^{H}[n-1] \longrightarrow \mathbf{Q}_{U}^{H}[n] \longrightarrow \boldsymbol{Q}_{X}^{H}[n] \longrightarrow 0
$$

where $Y$ is a principal divisor such that $U=X \backslash Y$ is smooth (restricting $X$ ), and $j: U \rightarrow X$.

Remarks. (i) If $\boldsymbol{Q}_{X}[n]$ is a perverse sheaf, $X$ is purely $n$-dimensional.

(ii) We say that $X$ is locally complete intersection, if $X$ is locally a closed subvariety of a smooth variety $Y$ such that $X=\cap_{1 \leq i \leq k} g_{i}^{-1}(0)_{\text {red }}$ for a regular sequence $g_{1}, \cdots, g_{k}$ (i.e. $k=\operatorname{dim} Y^{Y}-\operatorname{dim} X$ ). If $X$ is locally complete intersection of pure dimension $n$, then $Q_{X}[n]$ is a perverse sheaf.

(2.13) Lemma. Let $X$ be a quasiprojective variety, and $Z$ its closed subvariety which does not contain an irreducible component of $X$. Then there exists a locally principal divisor $Y$ of $X$ such that $Y \supset Z$ (and $X \backslash Y$ is dense in $X$ ).

Proof. We may assume $X$ is a closed subvariety of $\boldsymbol{P}^{n}$. If $X$ is irreducible, $Y$ is defined by a homogeneous polynomial of $m+1$ variables. In general, let $X=\cup_{i} X_{i}$ be the decomposition into irreducible components. Then there are homogeneous polynomials $P_{\imath}$ of degree $d_{\imath}$ such that $\cup_{\jmath \neq i} X, \cup Z \subset P_{i}^{-1}(0) \not \subset X_{\imath}$. We may assume $d_{i}=d_{j}$, and put $P=\sum_{i} P_{l}$.

Remarks. (i) If $X$ is locally complete intersection, so is $Y$.

(ii) We may assume $X \backslash Y$ smooth by replacing $Z$ with $Z \cup \operatorname{Sing} X$.

(2.14) Proof of (0.8). We first note that (0.9) is surjective for $p=1$ by definition of geometric level, and (0.10) is surjective for $p=0$, because (1.1.7) holds in $D^{b} \operatorname{MHM}(X)_{\mathrm{g} I \leqq m}^{\mathrm{g}}$ for $\operatorname{dim} X \leqq m$. We have the vanishing of negative extensions, because $\boldsymbol{Q}_{Y}^{H}\left[\operatorname{dim} Y^{Y}\right]$ is a mixed Hodge Module. So it is enough to show the first assertion. We proceed by induction on $\operatorname{dim} X$. The assertion is clear if $\operatorname{dim} X \leqq d$, because (1.1.7) holds for $D^{b} \operatorname{MHM}(X)_{\operatorname{gi} 1 \operatorname{dim} X}^{\mathrm{go}}$. So we may assume $\operatorname{dim} X>d$ and the assertion is proved for subvarieties of $X$ with smaller dimension. Let $\xi \in \operatorname{Ext}_{X, g 1 \leq n}^{-2 d}\left(\boldsymbol{Q}_{X}^{\prime \prime}, \boldsymbol{D}_{X}^{H}(-d)\right)$. Let $Z$ be a closed subvariety of $X$ with dimension $<\operatorname{dim} X$ such that $Y^{r}:=X \backslash Z$ is smooth of pure $\operatorname{dimension} \operatorname{dim} X$. If $X$ is quasiprojective, we may assume $Z$ is a locally principal divisor by (2.13). The restriction of $\xi$ to $Y$ is the image of $\xi^{\prime} \subseteq \operatorname{Ext}_{Y}^{2}, \operatorname{gl} \leqq \operatorname{dim} Y\left(Q_{Y}^{H}, Q_{Y}^{H}(p)\right)$ by hypothesis. Using a factorization of $\xi^{\prime}$ as in the proof of (2.9), we see that the restriction of $\xi^{\prime}$ to an open dense subvariety is zero by (2.1.3), and we may 
assume $\xi^{\prime}=0$ by replacing $Z$. Let $i: Z \rightarrow X, j: Y \rightarrow X$. We have a distinguished triangle

$$
\longrightarrow i_{*} \mathbb{D}_{Z}^{U} \longrightarrow \boldsymbol{D}_{X}^{H} \longrightarrow j_{*} \boldsymbol{D}_{Y}^{H} \longrightarrow \text {. }
$$

By adjunction for $i, j$ (cf. (2.2.v)) and (2.6), the assertion follows from the inductive hypothesis (applied to $Z$ ) and the surjectivity of $(0.9)$, using a morphism of the long exact sequences associated with (2.4.1).

(2.15) Proposition. We have naturally a cycle map (0.3) whose composition with (0.5) is the cycle map (0.4) in [17, II].

Proof. We may assume $n=\operatorname{dim} X$. By the same argument as in [loc. cit.], it is sufficient to show $\mathrm{cl}^{\mathrm{MH}}(\operatorname{div}(g))=0$ in $\operatorname{Ext}_{X, g 1 \leq \operatorname{dim} X}^{2}\left(\mathbb{Q}_{X}^{H}, \mathbb{Q}_{X}^{H}(1)\right)$ for a rational function $g$ on $X$ when $X$ is smooth projective. Then the assertion is reduced to [loc. cit.] by the injectivity of (0.5) in the divisor case, cf. (0.8).

\section{$\S 3$. Injectivity of Cycle Maps}

(3.1) Theorem. Let $X$ be a quasiprojective variety of pure dimension $n$, and $d \in N$. Assume $Q_{X}[n]$ is a perverse sheaf (resp. $X$ is locally complete intersection). Then the cycle map (0.3) for $d$ is injective, if the following conditions are satisfied:

(3.1.1) the cycle map (0.4) for $d$ is injective for any closed subvariety $Y$ of pure dimension $n-1$ such that $\mathbb{Q}_{Y}[n-1]$ is a perverse sheaf (resp. $Y$ is locally complete intersection),

(3.1.2) the cycle map (0.4) for $d+1$ is surjective for any purely n-dimensional smooth projective variety $X^{\prime}$.

Remark. Theorem (0.7) follows from (3.1) by induction on $n$.

We begin with some preliminaries.

(3.2) Yoneda extension. Let $A$ be an abelian category, and $M, N$ objects of $\mathcal{A}$. Then a Yoneda extension class $e \in{ }^{Y} \operatorname{Ext}_{\mathcal{H}}^{k}(M, N)$ is represented by a long exact sequence

$$
0 \longrightarrow N \longrightarrow L_{0} \longrightarrow \cdots \longrightarrow L_{k-1} \longrightarrow M \longrightarrow 0
$$

for $L_{i} \in \mathcal{A}$. This can be viewed as a resolution of $N$, and the morphism $M[-k] \rightarrow\left[L_{0} \rightarrow \cdots \rightarrow L_{k-1} \rightarrow M\right]$ induced by the identity on $M$ determines $e^{\prime} \in$ $\operatorname{Hom}_{D b A}(M[-k], N)$ which depends only on the Yoneda extension class Conversely, let $e^{\prime} \in \operatorname{Hom}_{D^{b} \mathfrak{A}}(M[-k], N)$ represented by a morphism

$$
M[-k] \longrightarrow L^{\prime}
$$


with $N \cong L^{\prime}$ a quasi-isomorphism which we can truncate between degree 0 and $k$ (i.e. $L_{\imath}^{\prime}=0$ for $i<0$ or $i>k$ ) using the filtration $\tau$. We define a complex $L$ by $L_{i}=L_{i}^{\prime}$ for $i<k-1, L_{\imath}=0$ for $i \geqq k$ and $L_{k-1}=\operatorname{Ker}\left(M \oplus L_{k-1}^{\prime} \rightarrow L_{k}^{\prime}\right)$. Then we have a natural quasi-isomorphism $L \simeq C\left(M[-k] \rightarrow L^{\prime}\right)$ with a morphism $L[1-k]$ $\rightarrow M$, which gives a long exact sequence (3.2.1) and determines a Yoneda extension class. These two correspondences are inverse of each other up to sign.

Let $e$ be a Yoneda extension class represented by (3.2.1) as above, and $e^{\prime} \in \operatorname{Hom}_{D b_{\mathcal{A}}}(M[-k], N)$ corresponding to $e$. Let $u: M \rightarrow M^{\prime}$ be a morphism in $A$ such that $e^{\prime}$ belongs to the image of $u^{*}: \operatorname{Hom}_{D^{b} A}\left(M^{\prime}[-k], N\right) \rightarrow$ $\operatorname{Hom}_{D b \_}(M[-k], N)$. Then there exist $L_{i}^{\prime}(0 \leqq i<k)$ and a morphism of long exact sequences

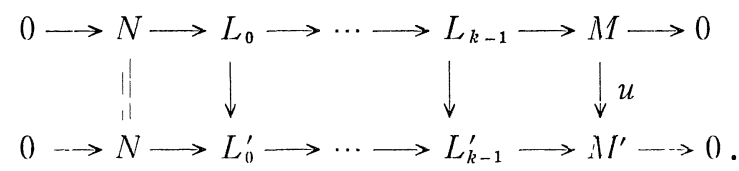

This follows from the definition of morphisms of $D^{b}$. 1 .

Remark. For a long exact sequence (3.2.1), let $V_{2}=\operatorname{Im}\left(L_{i-1} \rightarrow L_{i}\right)(0<i<k)$, $N_{0}=N, N_{k}=M$. We have a short exact sequence $0 \rightarrow N_{i} \rightarrow L_{i} \rightarrow N_{i+1} \rightarrow 0$ for $0 \leqq i<k$. We denote by $e_{i} \in \operatorname{Ext}_{\AA}^{1}\left(N_{i+1}, N_{\imath}\right)$ the corresponding extension class. Then $e^{\prime}$ coincides with the composition of $e_{i}$.

(3.3) Yoneda extension class associated with a cycle. Let $X$ be as in (3.1). Let $\zeta \in \mathrm{CH}_{d}(X)$, and $p=n-d$. By (2.13) there are closed subvarieties $X_{i}(1 \leqq i$ $\leqq p)$ of $X$ such that $\boldsymbol{Q}_{X_{2}}^{H}[n-i]$ is a perverse sheaf and supp $\zeta \subset X_{p}$. Then we have the cycle class $\xi_{i}=\operatorname{cl}^{\mathrm{MH}}(\zeta)$ in $\operatorname{Ext}_{X_{i}, \mathrm{go}_{\mathrm{o}}}^{-2 d}\left(\boldsymbol{Q}_{X_{i}}^{H}, \boldsymbol{D}_{X_{i}}^{H}(-d)\right)$ for $0 \leqq i \leqq p$, where $X_{0}=X$. By (2.12) and (2.2, v), $\xi_{i}$ is naturally defined in $\operatorname{Ext}_{X_{i}, \mathrm{~g} 1 \leqq n-i}^{-2 d}\left(\boldsymbol{Q}_{X_{i}}^{H}, \boldsymbol{D}_{X_{i}}^{H}(-d)\right)$. The restriction and Gysin morphisms $\boldsymbol{Q}_{X_{i-1}}^{I I} \rightarrow \boldsymbol{Q}_{X_{i}}^{H}$ and $\boldsymbol{D}_{X_{2}}^{H} \rightarrow \boldsymbol{D}_{X_{i-1}}^{H}$ determine extension classes

$$
\begin{aligned}
& e_{i} \in \operatorname{Ext}_{X_{i-1}, \mathrm{gl} \leqq n-\imath+1}^{1}\left(\boldsymbol{Q}_{X_{i-1}}^{H}[n-i+1], \boldsymbol{Q}_{X_{i}}^{I I}[n-i]\right), \\
& e_{i}^{\prime} \in \operatorname{Ext}_{X_{i-1}, g l \leqq n-\imath+1}^{1}\left(\boldsymbol{D}_{X_{i}}^{H}[i-n], \boldsymbol{D}_{X_{i-1}}^{H}[i-n-1]\right),
\end{aligned}
$$

(cf. (2.12.2)) such that $e_{i}^{\prime}{ }^{\circ} \xi_{i} \circ e_{i}=\xi_{i-1}$, and we get

$$
\left(\Pi_{1 \leqq i \leqq p} e_{i}^{\prime}\right) \circ \xi_{p^{\circ}}\left(\Pi_{1 \leqq i \leqq p} e_{i}\right)=\xi_{0} \quad \text { in } \operatorname{Ext}_{X, \mathbf{g l} \leqq n}^{2 p}\left(\boldsymbol{Q}_{X}^{H}[n], \boldsymbol{D}_{X}^{H}[-n](-d)\right) .
$$

This gives a representative of the Yoneda extension class corresponding to $\xi_{0}$. Let $X_{p}=\cup_{i} Z_{i}$ be the decomposition into irreducible components. By (1.1.7), $\xi_{p}$ belongs to

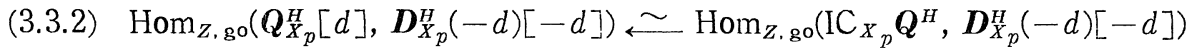

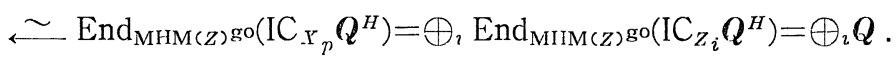


Its restriction to a generic point of $Z_{i}$ is the multiplication by a rational number $r_{i}$, and this number gives the coefficients of the cycle $\zeta$.

For $0 \leqq k \leqq p$, we define

$$
\eta_{k}=\left(\Pi_{1 \leqq i \leqq p} e_{i}^{\prime}\right) \circ \xi_{p} \circ\left(\Pi_{k<i \leqq p} e_{i}\right) \in \operatorname{Ext}_{X, \operatorname{gl}}^{2 p-k}\left(\boldsymbol{Q}_{X}^{H}[n-k], D_{X}^{H}[-n](-d)\right) .
$$

The following is a key proposition in the proof of (3.1).

(3.4) Proposition. With the above notation, let $\zeta \in \mathrm{CH}_{d}(X)$ for $d \leqq n-2$. If $\xi_{0}:=\mathrm{cl}^{\mathrm{MH}}(\zeta)$ is zero in $\operatorname{Ext}_{X, \mathrm{~g} 1 \leqq n}^{2 p}\left(\boldsymbol{Q}_{X}^{H}[n], \boldsymbol{D}_{X}^{H}[-n](-d)\right)$, there exist a locally principal divisor $Y$ on $X$ containing $X_{2}$, a projective morphism $\pi$ of a smooth variety $X^{\prime}$ of pure dimension $n$ onto $X$, and

$$
\begin{aligned}
& \eta \in \operatorname{Ext}_{X, \mathrm{~g} 1 \leqq n}^{2 p-2}\left(\pi_{*} Q_{X^{\prime}}^{H}[n](1), D_{X}^{H}[-n](-d)\right), \\
& \gamma \in \operatorname{Ext}_{X, \mathrm{~g} 1 \leqq n}^{1}\left(\mathbb{Q}_{Y}^{H}[n-1], \pi_{*} \mathbb{Q}_{X^{\prime}}^{H}[n](1)\right)
\end{aligned}
$$

such that $\eta \circ \gamma=\eta_{2} \circ f_{2}$ and $\gamma \circ f_{1}=0$, where $f_{1}: Q_{X}^{H}[n-1] \rightarrow Q_{Y}^{H}[n-1], f_{2}: Q_{Y}^{H}[n-2]$ $\rightarrow Q_{X_{2}}^{H}[n-2]$ are natural morphisms.

Proof. Let $j: U=X \backslash X_{1} \rightarrow X$. By the long exact sequence associated with $0 \rightarrow Q_{X_{1}}^{H}[n-1] \rightarrow j_{!} \boldsymbol{Q}_{U}^{H}[n] \rightarrow Q_{X}^{H}[n] \rightarrow 0$, the condition $\xi_{0}=\eta_{1} \circ e_{1}=0$ is equivalent to that $\eta_{1}$ belongs to the image of

$$
\operatorname{Ext}_{X, \operatorname{gl}}^{2 p-1}\left(j, \boldsymbol{Q}_{U}^{H}[n], \boldsymbol{D}_{X}^{H}[-n](-d)\right) \longrightarrow \operatorname{Ext}_{X, g_{1}^{2}}^{2 p-1}\left(\boldsymbol{Q}_{X_{1}}^{H}[n-1], \boldsymbol{D}_{X}^{H}[-n](-d)\right) .
$$

By (3.2) we have a morphism of long exact sequences (3.2.3) in $\operatorname{MHM}(X)$ go such that $M=\boldsymbol{Q}_{X_{1}}^{H}[n-1], M^{\prime}=j_{!} \boldsymbol{Q}_{U}^{H}[n], \quad N=\mathbb{D}_{X}^{H}[-n](-d), k=2 p-1$, where the first long exact sequence corresponds to (3.3.1) as in Remark after (3.2). Put $N^{\prime}=L_{k-1}^{\prime}, \quad N^{\prime \prime}=\operatorname{Im}\left(L_{k-2}^{\prime} \rightarrow L_{k-1}^{\prime}\right)$. Then the morphism of long exact sequences induces

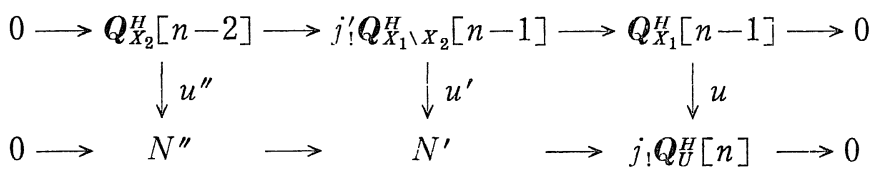

where $j^{\prime}: X_{1} \backslash X_{2} \rightarrow X_{1}$. We denote by

$$
\eta_{1}^{\prime} \in \operatorname{Ext}_{X, \operatorname{gl} 1 \leq n}^{2 p-1}\left(j ! \mathbb{Q}_{U}^{H}[n], \boldsymbol{D}_{X}^{H}[-n](-d)\right), \quad \eta_{2}^{\prime} \in \operatorname{Ext}_{X, \mathbf{g}_{1} \leq n}^{2 p-2}\left(N^{\prime \prime}, \mathbb{D}_{X}^{H}[-n](-d)\right)
$$

the extension classes induced by the second long exact sequence of (3.2.3) so that

$$
\eta_{1}^{\prime} \circ u=\eta_{1}, \quad \eta_{2}^{\prime} \circ u^{\prime \prime}=\eta_{2}, \quad \eta_{2}^{\prime} \circ e=\eta_{1}^{\prime},
$$

where $e \in \operatorname{Ext}_{X, g 1 \unlhd n}^{1}\left(j, Q_{U}^{H}[n], N^{\prime \prime}\right)$ is the extension class associated with the second exact sequence of (3.4.1). By [17, II, (4.5)], the composition of $\eta_{2}^{\prime}$ with $W_{n-3} N^{\prime \prime}$ $\rightarrow N^{\prime \prime}$ is zero, because $\boldsymbol{D}_{X}^{H}[-n](-d)$ has weights $\geqq-n+2 d$. So $\eta_{2}^{\prime}$ is factorized by the projection $N^{\prime \prime} \rightarrow N^{\prime \prime} / W_{n_{-3}} N^{\prime \prime}$. Replacing $N^{\prime \prime}$ by $N^{\prime \prime} / W_{n-3} N^{\prime \prime}$ (and $e, u^{\prime \prime}$ 
by their composition with the projection), we may assume

$$
\text { wt } N^{\prime \prime} \geqq n-2 \text {. }
$$

We will modify $N^{\prime}, N^{\prime \prime}$ so that they become as simple as possible. Using (3.4.1), we can check

(3.4.3) If we have a morphism $N^{\prime} \rightarrow L$ such that its composition with $u^{\prime}$ is zero and the composition $N^{\prime \prime} \rightarrow N^{\prime} \rightarrow L$ is surjective, then we may replace $N^{\prime}, N^{\prime \prime}$ by $\operatorname{Ker}\left(N^{\prime} \rightarrow L\right), \operatorname{Ker}\left(N^{\prime \prime} \rightarrow L\right)$,

because $u^{\prime}, u^{\prime \prime}$ is factorized by $\operatorname{Ker}\left(N^{\prime} \rightarrow L\right), \operatorname{Ker}\left(N^{\prime \prime} \rightarrow L\right)$. Then we may assume

$$
\text { wt } N^{\prime \prime} \leqq n-1 \text {, }
$$

because wt $j_{!}^{\prime} \boldsymbol{Q}_{X_{1} \backslash X_{2}}^{H}[n-1] \leqq n-1$ and wt $j_{!} \boldsymbol{Q}_{U}^{H}[n] \leqq n$. In fact, we may assume first

$$
\text { wt } N^{\prime} \leqq n,
$$

and then (3.4.4) using semisimplicity of $\mathrm{Gr}_{n}^{\mathrm{T}} N^{\prime}$ which induces a splitting of $\mathrm{Gr}_{n}^{\mathrm{W}}$ of the second exact sequence of (3.4.1). Let $e^{\prime}$ be the composition of $e$ with $N^{\prime \prime} \rightarrow \operatorname{Gr}_{n-1}^{W W} N^{\prime \prime}$. Since wt $Q_{X_{2}}^{H}[n-2] \leqq n-2$, the composition of $u$ with $e^{\prime}$ is zero by the commutativity of (3.4.1), and $e^{\prime}$ is the composition of $j_{:} \boldsymbol{Q}_{U}^{H}[n] \rightarrow \boldsymbol{Q}_{X}^{H}[n]$ with

$$
e^{\prime \prime} \in \operatorname{Ext}_{X, \mathrm{gl} n n}^{1}\left(\boldsymbol{Q}_{X}^{I J}[n], \mathrm{Gr}_{n-1}^{W} N^{\prime \prime}\right) .
$$

Let $\operatorname{Gr}_{n-1}^{1 W} N^{\prime \prime}=\bigoplus_{Z}\left(\mathrm{Gr}_{n-1}^{W V} N^{\prime \prime}\right)_{Z}$ be the decomposition by strict support. Then

$$
\left(\mathrm{Gr}_{n-1}^{W V} N^{\prime \prime}\right)_{Z}=0 \quad \text { for } \operatorname{dim} Z=n,
$$

because $\operatorname{Gr}_{n-1}^{1 W} N^{\prime \prime}$ has level $<n$ by (2.1.3). The composition of $e^{\prime \prime}$ with the projection $\operatorname{Gr}_{n-1}^{W} N^{\prime \prime} \rightarrow\left(\mathrm{Gr}_{n-1}^{W I} N^{\prime \prime}\right)_{Z}$ is factorized by $Q_{X}^{H}[n-1] \rightarrow Q_{Z}^{I I}[n-1]$, and is zero for $\operatorname{dim} Z<n-1$, because $H^{2} Q_{Z}^{H}=0$ for $i>\operatorname{dim} Z$. If $\operatorname{dim} Z=n-1$, the image of the morphism $Q_{Z}^{I}[n-1] \rightarrow\left(\mathrm{Gr}_{n-1}^{I} N^{\prime \prime}\right)_{Z}$ is either $\mathrm{IC}_{Z} Q^{I I}$ or zero. So we may assume

$$
\operatorname{Gr}_{n-1}^{\prime \prime} N^{\prime \prime}=\oplus_{2} \mathrm{IC}_{Y_{2}} Q^{I I}
$$

by (3.4.3) so that $e^{\prime \prime}$ is identified with the natural morphism

$$
\boldsymbol{Q}_{X}^{I I}[n-1] \longrightarrow \oplus_{\imath} Q_{Y_{\imath}}^{H}[n-1] \longrightarrow \oplus_{\imath} \mathrm{IC}_{Y_{i}} \boldsymbol{Q}^{H},
$$

where $Y_{i}$ are irreducible closed subvarieties of $X$ with codimension one. In fact, let $\mathrm{Gr}_{n-1}^{I V} N^{\prime \prime}=L \oplus L^{\prime}$ be a decomposition such that $e^{\prime \prime}$ (and hence $e^{\prime}$ ) is factorized by $L \rightarrow \mathrm{Gr}_{n-1}^{W} N^{\prime \prime}$. Considering the extension class $e$ modulo $W_{n-2} N^{\prime}+\operatorname{Im} u^{\prime}$, we get a splitting of $L^{\prime} \rightarrow N^{\prime} / W_{n-2} N^{\prime}+\operatorname{Im} u^{\prime}$, and we can apply (3.4.3) to the projection $N^{\prime} \rightarrow N^{\prime} / W_{n-2} N^{\prime}+\operatorname{Im} u^{\prime} \rightarrow L^{\prime}$. Let

Then we have

$$
A=W_{n-2} N^{\prime \prime}+\operatorname{Im} u^{\prime} \text {. }
$$




$$
\operatorname{Im}\left(A \rightarrow N^{\prime} / N^{\prime \prime}=j: Q_{U}^{U}[n]\right)=\operatorname{Im} u=Q_{X_{1}}^{H}[n-1]
$$

with a short exact sequence

$$
0 \longrightarrow W_{n-2} N^{\prime \prime} \longrightarrow A \longrightarrow Q_{X_{1}}^{H}[n-1] \longrightarrow 0,
$$

because $N^{\prime \prime} \cap \operatorname{Im} u^{\prime}=\operatorname{Im} u^{\prime \prime}$ by the injectivity of $u$ in (3.4.1), cf. (2.12.2). This implies also a short exact sequence

$$
0 \longrightarrow \operatorname{Im} u^{\prime} \longrightarrow A \longrightarrow W_{n-2} N^{\prime \prime} / \operatorname{Im} i i^{\prime \prime} \longrightarrow 0
$$

which splits by the semisimplicity of $W_{n-2} N^{\prime \prime}$ (cf. (3.4.2)), i.e..

$$
A=\operatorname{Im} u^{\prime} \oplus A_{1}
$$

for a subobject $A_{1}$ of $W_{n-2} N^{\prime \prime}$.

Let $Y$ be a locally principal divisor of $X$ containing the $Y_{2}$. We have a natural surjective morphism $v_{0}: Q_{Y}^{H}[n-1] \rightarrow \operatorname{Gr}_{n-1}^{W} N^{\prime \prime}$, which factorizes $e^{\prime \prime}$ naturally, cf. (3.4.5). So we get a morphism of short exact sequences

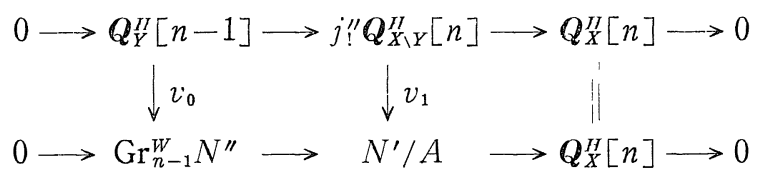

where $j^{\prime \prime}: X \backslash Y \rightarrow X$, and the extension class of the second exact sequence is $e^{\prime \prime}$. Then we can modify $N^{\prime}$ by replacing $\operatorname{Gr}_{n-1}^{V} N^{\prime \prime}$ with $\mathbb{Q}_{Y}^{H}[n-1]$, and get a mixed Hodge Module $\bar{N}^{\prime}$ with a morphism of short exact sequences

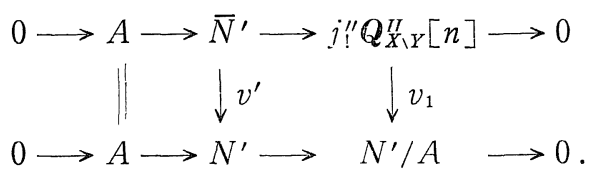

(In fact, $\bar{N}^{\prime}$ is defined by it.) Since $u^{\prime}$ is factorized by $A, u^{\prime}$ is naturally factorized by $v^{\prime}$. This induces a factorization of the morphism (3.4.1) by

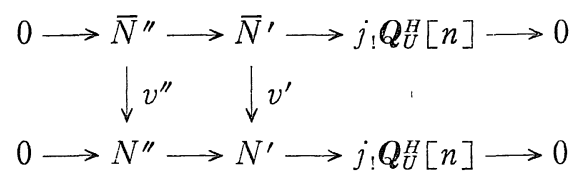

where $\bar{N}^{\prime \prime}$ is defined by the first exact sequence. In particular, we have $u^{\prime \prime}=$ $v^{\prime \prime} \circ w^{\prime \prime}$ for $w^{\prime \prime}: Q_{X_{2}}^{H}[n-2] \rightarrow \bar{N}^{\prime \prime}$ so that

$$
\eta_{2}=\eta_{2}^{\prime \prime} \circ w^{\prime \prime}
$$

with $\eta_{2}^{\prime \prime}=\eta_{2}^{\prime} \circ v^{\prime \prime}$. We define a filtration $G$ of $\bar{N}^{\prime}$ by $G_{-1} \bar{N}^{\prime}=0, G_{0} \bar{N}^{\prime}=A \cap \bar{N}^{\prime \prime}$ $\left(=A \cap N^{\prime \prime}\right), \quad G_{1} \bar{N}^{\prime}=A+\bar{N}^{\prime \prime}, \quad G_{2} \bar{N}^{\prime}=\bar{N}^{\prime} . \quad$ Let $A_{0}=\operatorname{Im} u^{\prime \prime}, \quad B_{0}=\boldsymbol{Q}_{X_{1}}^{H}[n-1], \quad B_{1}=$ $\mathbb{Q}_{Y}^{H}[n-1], C=\mathbb{Q}_{X}^{H}[n]$. Then 


$$
\mathrm{Gr}_{0}^{G} \bar{N}^{\prime}=A_{0} \oplus A_{1}, \quad \mathrm{Gr}_{1}^{G} \bar{N}^{\prime}=B_{0} \oplus B_{1}, \quad \operatorname{Gr}_{2}^{G} \bar{N}^{\prime}=C,
$$

because $G$ is defined by the convolution of the two filtrations defined by the first exact sequences of (3.4.7-8), and $A \cap \bar{N}^{\prime \prime}=\operatorname{Ker}\left(A \rightarrow j_{!} \boldsymbol{Q}_{U}^{H}[n]\right)$, etc. Let $\beta_{\imath} \in$ $\operatorname{Ext}^{1}\left(C, B_{i}\right), \alpha_{\jmath i} \in \operatorname{Ext}^{1}\left(B_{i}, A_{\jmath}\right)$ denote the extension classes induced by $\bar{N}^{\prime}$. Since the composition of the extension class of $\operatorname{Gr}_{0}^{G} \bar{N}^{\prime}, \operatorname{Gr}_{1}^{G} \bar{N}^{\prime}$ and that of $\operatorname{Gr}_{1}^{G} \bar{N}^{\prime}$, $\operatorname{Gr}_{2}^{G} \bar{N}^{\prime}$ is zero, we get

$$
\alpha_{j 0} \circ \beta_{0}+\alpha_{\jmath 1} \circ \beta_{1}=0 \quad(j=0,1) .
$$

By definition, $\beta_{\imath}(i=0,1)$ and $\alpha_{00}$ are natural morphisms. Then $\alpha_{01}$ is also a natural morphism up to sign by (3.4.10), using adjunction. By (3.4.6), we get $\alpha_{10}=0$, because $A$ is the extension defined by $\left(\alpha_{00}, \alpha_{10}\right)$. So

$$
\alpha_{11} \circ \beta_{1}=0
$$

by (3.4.10). Let $\lambda_{\imath} \subseteq \operatorname{Ext}_{X, \text { gl }}^{2 p-2}\left(A_{\imath}, D_{X}^{J I}[-n](-d)\right)$ be the composition of the inclusion $A_{2} \rightarrow \bar{N}^{\prime \prime}$ with $\eta_{2}^{\prime \prime}$. By the long exact sequence associated with $0 \rightarrow A_{0} \oplus A_{1}$ $\rightarrow \bar{N}^{\prime \prime} \rightarrow B_{1} \rightarrow 0$, we get

$$
\lambda_{0} \circ \alpha_{01}+\lambda_{1} \circ \alpha_{11}=0 \text {. }
$$

Applying (3.4.3) to $\bar{N}^{\prime}$, we may assume further

(3.4.13) $A_{1}$ has strict support of dimension $n$.

In fact, let $A_{1}=\bigoplus_{Z}\left(A_{1}\right)_{Z}$ be the decomposition by strict support. Then, considering $\bar{N}^{\prime} / \operatorname{Im} u^{\prime}$, it is enough to show that the composition of $j_{!}^{\prime \prime} \boldsymbol{Q}_{X_{Y}}^{H}[n]=$ $\bar{N}^{\prime} / A \rightarrow A_{1}[1]$ with the projection $A_{1}[1] \rightarrow\left(A_{1}\right)_{Z}[1]$ is zero for $d_{Z} \neq n$. Here we can restrict to $X \backslash Y$ by adjunction for $j^{\prime \prime}$, and we may replace $j_{!}^{\prime \prime} \boldsymbol{Q}_{X \backslash Y}^{H}[n]$ by $\boldsymbol{Q}_{X}^{H}[n]$. The case $d_{Z}<n-1$ follows from [16, (4.5.6)] using adjunction for $Z \rightarrow X$. The case $d_{Z}=n-1$ is reduced to $\operatorname{Hom}\left(Q_{Z}^{H}[n-1],\left(A_{1}\right)_{Z}\right)=$ Hom $\left(H^{n-1} Q_{Z}^{H},\left(A_{1}\right)_{Z}\right)=0$ by the same argument, and we can check it by restricting to a smooth open subvariety of $Z$, because $A_{1}$ is pure of weight $n-2$.

Since the geometric level of $A_{1}$ is $n, A_{1}$ is a direct factor of $H^{0} \pi_{*}\left(\boldsymbol{Q}_{X^{\prime}}^{I}[n]\right)(1)$ for a proper morphism $\pi$ of a smooth projective variety $X^{\prime}$ of pure dimension $n$ onto $X$. By the decomposition theorem (cf. (2.2.1)), $A_{1}$ is a direct factor of $\pi_{*} Q_{X^{\prime}}^{\eta \eta}[n](1)$. Let $\gamma, \eta$ be the composition of $\alpha_{11}, \lambda_{1}$ with the associated morphisms $A_{1} \rightarrow \pi_{*} \boldsymbol{Q}_{X^{\prime}}^{H}[n](1), \pi_{*} \boldsymbol{Q}_{X^{\prime}}^{H}[n](1) \rightarrow A_{1}$ respectively. Then (3.4.11) and (3.4.12) hold with $\alpha_{11}, \lambda_{1}$ replaced by $\gamma, \eta$. Since $w^{\prime \prime}$ is the composition of natural morphisms $\boldsymbol{Q}_{X_{2}}^{H}[n-2] \rightarrow A_{0} \rightarrow \bar{N}^{\prime \prime}, \eta_{2}$ is the composition of $\boldsymbol{Q}_{X_{2}}^{H}[n-2] \rightarrow A_{0}$ with $\lambda_{0}$, and we get $\lambda_{0} \circ \alpha_{01}=-\eta_{2} \circ f_{2}$. So the assertion follows, because $\beta_{1}=f_{1}$.

(3.5) Lemma. Let $X$ be a smooth quasi-projective variety of pure dimension $n$, and $Y^{r}$ a (reduced) divisor on $X$. Let $Y^{r}=\cup_{2} Y^{r}{ }_{2}$ be the decomposition into irreducible components. Lel $\iota_{\imath}$ be the composition of natural morphisms 


$$
\boldsymbol{Q}_{Y}^{H} \longrightarrow \boldsymbol{Q}_{Y_{\imath}}^{H} \longrightarrow \boldsymbol{D}_{Y_{\imath}}^{H}(1-n)[2-2 n] \longrightarrow \boldsymbol{D}_{X}^{H}(1-n)[2-2 n]=\mathbf{Q}_{X}^{H}(1)[2] .
$$

Then the ¿ $\iota_{\text {form }}$ a basis of a $\mathbf{Q}$-vector space $\operatorname{Ext}_{X, g_{0}}^{2}\left(\mathbb{Q}_{Y}^{I I}, \mathbb{Q}_{X}^{H}(1)\right)$.

Proof. By the adjunction for $i: Y \rightarrow X$, the assertion is reduced to (1.1.7).

(3.6) Proposition. With the above notation, let $\zeta \Subset \mathrm{CH}_{d}(X)_{Q}$, and $\zeta^{\prime}=$ $\sum_{i} r_{2}\left(\zeta \cdot\left[Y_{i}\right]\right) \in \mathrm{CH}_{d-1}(Y)_{\boldsymbol{Q}}$ for $r_{i} \in \mathbf{Q}$. Then the composition of $\sum_{2} r_{i} \iota_{2}: Q_{Y}^{H} \rightarrow$ $Q_{X}^{H}(1)[2]$ with $\mathrm{cl}^{\mathrm{MH}}(\zeta)$ coincides with the composition of $\mathrm{cl}^{\mathrm{NH}}\left(\zeta^{\prime}\right)$ with the Gysin morphism $\boldsymbol{D}_{Y}^{H} \rightarrow \boldsymbol{D}_{X}^{H}$.

Proof. We may assume $I^{r}$ irreducible, $r=1$, and $\zeta$ is represented by an irreducible closed subvariety $Z$ which intersects properly with $Y$ by the classical moving lemma. Let $Z^{\prime}=Z \cap Y$. Using the adjunction for the inclusion $i: Y \rightarrow X$, we get a commutative diagram

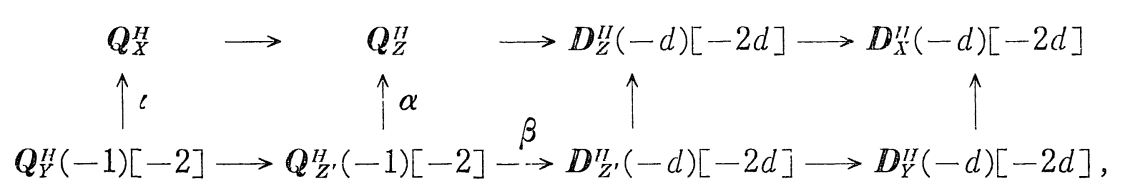

where $\iota$ is as in (3.5.1), $\alpha$ and $\beta$ are induced by the adjunction and determined uniquely by the commutativity, and the other morphisms are natural ones. By (1.1.7), $\beta$ is uniquely determined by its restriction to a generic point of each irreducible component of $Z^{\prime}$, and it is enough to show that it is the multiplication by the intersection multiplicity. In particular, it is enough to consider the underlying $\boldsymbol{Q}$-complexes. So may assume $Z$ is a curve and $d=1$ by intersecting with a generic smooth subvariety of $X$ with codimension $d-1$, which intersects transversally with $Z$. Let $g$ be a (reduced) defining equation of $Y$. We can check that $\iota$ is obtained by the composition of a natural morphism $i^{*} \rightarrow \phi_{g}$ and its dual $\phi_{g} \rightarrow i^{!}(1)[2]$ (by restricting to a generic point of $Y$ ), and $\beta: \boldsymbol{Q}_{Z^{\prime}} \rightarrow \boldsymbol{D}_{Z^{\prime}}$ coincides with the composition of $\boldsymbol{Q}_{Z^{\prime}}=i^{*} \boldsymbol{Q}_{Z} \rightarrow \phi_{g} \boldsymbol{Q}_{Z}$ with its dual. Then we get the assertion, because $\psi_{g} \boldsymbol{Q}_{Z} \cong \Gamma\left(Z \cap g^{-1}(t), \boldsymbol{Q}\right) \stackrel{m}{\oplus} \boldsymbol{Q}$ for $t \neq 0$ sufficiently small, where $m$ is the intersection multiplicity.

(3.7) Proof of (3.1). The assertion is clear by (1.1.7) if $d=n$, and follows from the next proposition if $d=n-1$. So we may assume $d \leqq n-2$. Let $\zeta \in$ $\mathrm{CH}_{d}(X)_{Q}$ such that $\xi_{0}=\operatorname{cl}^{\mathrm{MH}}(\zeta)=0$. By (3.4) we have $Y, X^{\prime}, \gamma, \eta$ as in (3.4). Let

$$
\boldsymbol{\gamma}^{\prime} \in \operatorname{Ext}_{X^{\prime}, g_{0}}^{1}\left(\boldsymbol{Q}_{Y^{\prime}}^{H}[n-1], \boldsymbol{Q}_{X^{\prime}}^{H}[n](1)\right), \quad \eta^{\prime} \in \operatorname{Ext}_{X^{\prime}, g_{0}}^{p p-2}\left(\boldsymbol{Q}_{X^{\prime}}^{H},[n](1), \mathbb{D}_{X^{\prime}}^{H}[-n](-d)\right)
$$

be the elements corresponding to $\gamma, \eta$ by adjunction, where $Y^{\prime}=\pi^{-1}(Y)$. Here $\gamma, \eta$ are considered in the extension group defined in $D^{b} \mathrm{MHM}(X)^{\circ \circ}$. Let $Y^{\prime}=$ $\cup_{i} Y_{\imath}^{\prime}$ be the decomposition into irreducible components. By (3.5), we have $\gamma^{\prime}=$ $\sum_{i} r_{\imath} \iota_{\imath}$ for $r_{\imath} \in \boldsymbol{Q}$, where $\iota_{\imath}$ is defined by (3.5.1) with $X, \gamma^{\gamma}, Y_{\imath}$ replaced by $X^{\prime}$, 
$I^{\prime \prime}, I^{\prime \prime}$, By (1.4) and (3.1.2), there exists $\zeta^{\prime} \in \mathrm{CH}_{d+1}\left(X^{\prime}\right)_{Q}$ such that $\eta^{\prime}=\mathrm{cl}^{\mathrm{MII}}\left(\zeta^{\prime}\right)$. Let $\zeta^{\prime \prime}=\sum_{\imath} r_{\imath}\left(\zeta^{\prime} \cdot\left[Y_{i}\right]\right) \in \mathrm{CH}_{d}\left(Y^{\prime}\right)_{Q}$, and $\eta^{\prime \prime}=\mathrm{cl}^{\mathrm{MH}}\left(\zeta^{\prime \prime}\right)$. By (3.6), $\eta^{\prime} \circ \gamma^{\prime}$ coincides with the composition of $\eta^{\prime \prime}$ with the Gysin morphism $\boldsymbol{D}_{Y^{\prime}}^{H} \rightarrow \boldsymbol{D}_{X^{\prime}}^{H}$. Since $\eta, \gamma$ are the composition of $\pi_{*} \eta^{\prime}, \pi_{*} \gamma^{\prime}$ with the Gysin and restriction morphisms $\pi_{*} \boldsymbol{D}_{X^{\prime}}^{U}$ $\rightarrow D_{X}^{I \prime}, \quad Q_{Y}^{H} \rightarrow \pi_{*} \boldsymbol{Q}_{Y^{\prime}}^{I I}$ respectively, $\eta \circ \gamma$ coincides with the composition of $\pi_{*} \eta^{\prime \prime}$ with the Gysin morphism $\boldsymbol{D}_{Y}^{H} \rightarrow \boldsymbol{D}_{X}^{H}$ by definition of direct image (cf. [17, II, (2.3.6)]) using the commutative diagram

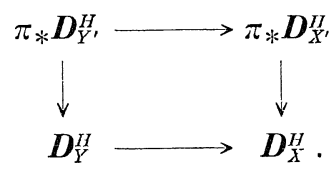

Let $\bar{\zeta} \in \mathrm{CH}_{d}(Y)_{Q}$ such that $i_{*} \bar{\zeta}=\zeta$ for $i: Y \rightarrow X$. Then $\eta_{2^{\circ}} f_{2}$ coincides with the composition of $\bar{\xi}=\mathrm{cl}^{\mathrm{NI}}(\bar{\zeta})$ with the Gysin morphism $\boldsymbol{D}_{Y}^{I I} \rightarrow \boldsymbol{D}_{X}^{H}$. By adjunction, $\eta \circ \gamma=\eta_{2} \circ f_{2}$ implies $\pi_{*} \eta^{\prime \prime}=\bar{\xi}$. Since $\pi_{*} \eta^{\prime \prime}=\operatorname{cl}^{N 111}\left(\pi_{*} \zeta^{\prime \prime}\right)$ by $[17$, II, (2.4)], we get $\pi_{*} \zeta^{\prime \prime}=\bar{\zeta}$ in $\mathrm{CH}_{d}(Y)_{Q}$ by (3.1.1). So it is sufficient to show $i_{*}^{\prime} \zeta^{\prime \prime}=0$ in $\mathrm{CH}_{d}\left(X^{\prime}\right)_{Q}$ where $i^{\prime}: Y^{\prime} \rightarrow X^{\prime}$. By definition, $i_{*}^{\prime} \zeta^{\prime \prime}$ is the intersection of $\zeta^{\prime}$ with $\sum_{\imath} r_{i}\left[Y_{\imath}\right]$, and it is enough to show $\zeta_{1}=\sum_{i} r_{2}\left[Y_{2}\right]=0$ in $\mathrm{CH}^{1}\left(X^{\prime}\right)_{Q}$. We have $\operatorname{cl}^{\mathrm{MH}}\left(\zeta_{1}\right)=\gamma^{\prime} \circ f_{1}^{\prime}$ by definition of $\iota_{2}$, where $f_{1}^{\prime}: \boldsymbol{Q}_{X^{\prime}}^{H} \rightarrow \boldsymbol{Q}_{Y^{\prime}}^{H}$ is the natural morphism. Using the dual diagram of (3.7.1), $\gamma^{\prime} \circ f_{1}^{\prime}$ corresponds to $\gamma_{\circ} f_{1}=0$ by adjunction. So we get $\mathrm{cl}^{\mathrm{MH}}\left(\zeta_{1}\right)=0$, and the assertion is reduced to the case $d=n-1$ and follows from the next proposition.

(3.8) Proposition. Let $X$ be an n-dimensional variety. Then we have a cycle map

$$
\operatorname{cl}^{\mathrm{II}}: \mathrm{CH}_{n-1}(X)_{Q} \longrightarrow \operatorname{Ext}_{X}^{2-2 n}\left(\boldsymbol{Q}_{X}^{I}, \boldsymbol{D}_{X}^{H}(1-n)\right),
$$

(cf. $[16][17, \mathrm{I}]$ ), and it is bijective, where $\operatorname{Ext}_{X}^{2}=\operatorname{Ext}_{D b \operatorname{MuM}(X)}^{2}$. Moreover, $(0.3-4)$ are injective for $d=n-1$.

Proof. It is enough to show the first assertion, because (0.3-4) are factorized by (3.8.1). If $X$ is smooth, the assertion is proved in [17, $\mathrm{I},(3.4)]$. Here $X$ may be not necessarily pure dimensional, because the $(n-1)$-dimensional case is clear. In general, let $\pi: X^{\prime} \rightarrow X$ be the normalization. Let $Z$ be a closed subvariety of $X$ such that $\operatorname{dim} Z \leqq n-2$. Let $U=X \backslash Z$. Then the natural morphisms

$$
\begin{aligned}
\mathrm{CH}_{n-1}(X)_{Q} & \longrightarrow \mathrm{CH}_{n-1}(U)_{Q} \\
\operatorname{Ext}_{X}^{2-2 n}\left(\boldsymbol{Q}_{X}^{H}, \boldsymbol{D}_{X}^{H}(1-n)\right) & \longrightarrow \operatorname{Ext}_{U}^{2-2 n}\left(\boldsymbol{Q}_{U}^{I}, \boldsymbol{D}_{U}^{H}(1-n)\right)
\end{aligned}
$$

are isomorphisms. In fact, the first isomorphism is clear. For the second, we use a long exact sequence associated with a triangle like (2.14.1) together with the adjunction isomorphisms as in $(2.2, \mathrm{v})$. Then the assertion follows from 
$[16,(4.5 .6)]$ and $[17, \mathrm{II},(4.5)]$.

We denote by $X_{k}$ the union of the irreducible components of dimension $\leqq k$. Let $Y=X_{n-1} \cup \operatorname{Sing} X, Z^{\prime}=\operatorname{Sing} Y^{\prime} \cup Y_{n-2} \cup \pi\left(\operatorname{Sing} X^{\prime}\right)$. Then $\operatorname{dim} Z^{\prime} \leqq n-2$. We take $Z$ containing $Z^{\prime}$. Replacing $X$ by $U$, we may assume $X^{\prime}$ smooth and $X_{n-1} \cup$ Sing $X$ smooth of pure dimension $n-1$. We may assume also $X$ is purely $n$-dimensional, because $X_{n-1}$ does not intersect with an $n$-dimensional irreducible component if we delete the intersection.

Let $Y=\operatorname{Sing} X$, and $Y^{\prime}=\pi^{-1}(Y)$. We may also assume that $\pi^{\prime}=\pi \mid Y^{\prime}: Y^{\prime} \rightarrow Y$ is étale, because we can take $Z$ containing $\pi^{\prime}\left(\operatorname{Sing} \pi^{\prime}\right)$. We have a distinguished triangle

$$
\longrightarrow \operatorname{Ker}\left(\pi_{*}^{\prime} \boldsymbol{D}_{Y^{\prime}}^{I I} \rightarrow \boldsymbol{D}_{Y}^{H}\right) \longrightarrow \pi_{*} \boldsymbol{D}_{X^{\prime}}^{I I} \longrightarrow \boldsymbol{D}_{X}^{I} \longrightarrow
$$

using base change by $i: Y \rightarrow X$, and we get the commutative diagram

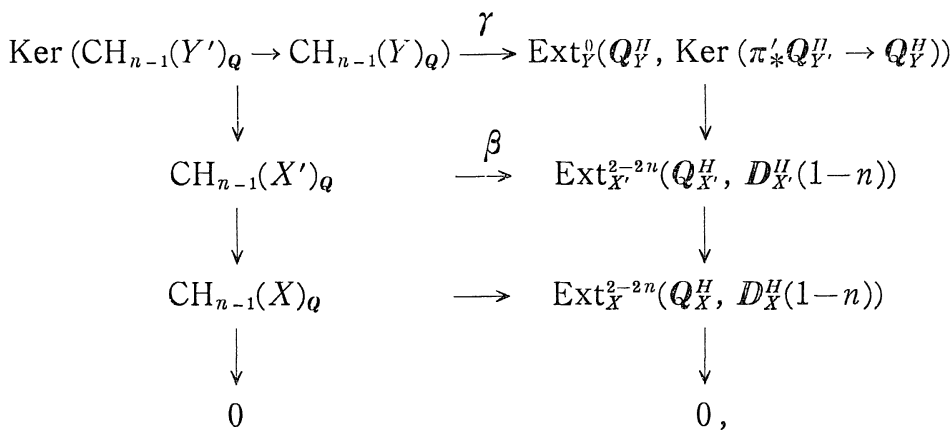

where the vertical sequences are exact. By $[17, I,(3.4)], \beta$ is bijective, and it is enough to show the bijectivity of $\gamma$. Here we may assume $Y$ connected by restricting to each connected component. Let $k$ be the number of the connected components of $\pi^{-1}(Y)$. Then the source and the target of $\gamma$ are both $Q$-vector spaces of dimension $k-1$ and we get the bijectivity of $\gamma$, where we use the adjunction for $\pi^{\prime}$ together with a splitting of the surjective morphism $\pi_{*}^{\prime} Q_{Y^{\prime}}^{I I}$ $\rightarrow \boldsymbol{Q}_{Y}^{H}$.

(3.9) Theorem. Let $X$ be an n-dimensional variety. Then (0.3) for $d=n-1$ is bijective, and

$$
\mathrm{cl}^{\mathrm{MH}}: \mathrm{CH}_{n-1}(X)_{Q} \longrightarrow \operatorname{Ext}_{X, \mathbf{g l} \leq n+1}^{2-2 n}\left(\boldsymbol{Q}_{X}^{H}, \boldsymbol{D}_{X}^{H}(1-n)\right),
$$

for $d=n-1$ is also bijective.

Proof. By (3.8) it is enough to show the surjectivity. Let $\xi \in$ $\operatorname{Ext}_{X, \mathbf{g} 1 \leq n+1}^{2-2 n}\left(\boldsymbol{Q}_{X}^{H}, \boldsymbol{D}_{X}^{H}(1-n)\right)$. By (1.1.7), it is enough to find an open subvariety $U$ of $X$ such that $\operatorname{dim} X \backslash U<n$ and the restriction of $\xi$ to $U$ is zero. Then the assertion for (0.3) is clear by (2.1.3). For (3.9.1), the assertion follows 
from an argument similar to the proof of (2.10). In fact, we may assume $X$ smooth, and $\xi=\xi_{1} \xi_{2}$ for $\xi_{\jmath} \in \operatorname{Ext}_{X, \mathrm{~g} 1 \leqq n+1}^{1}\left(M_{\jmath}, M_{\jmath-1}\right)=\operatorname{Ext}_{X, \mathrm{go}}^{1}\left(M_{\jmath}, M_{\jmath-1}\right)$ with $M_{0}=$ $\boldsymbol{Q}_{X}^{H}[n], M_{2}=\boldsymbol{Q}_{X}^{I}[n](-1)$, and $M_{1}=H^{0} \pi_{*} \boldsymbol{Q}_{Y}^{H}[n+1]$ for a smooth projective morphism $\pi: Y \rightarrow X$ of relative dimension 1 . Then it is enough to show that $\xi_{\text {, are }}$ induced by divisors in $Y=I^{Y} \times_{X} X$, because we can choose the divisors so that they do not intersect by restricting $X$. Here we may consider $\xi$, in $\operatorname{MHM}(X)$ by the injectivity of $\operatorname{Ext}_{X, \text { go }}^{1}\left(M_{\jmath}, M_{\jmath-1}\right) \rightarrow \operatorname{Ext}_{X}^{1}\left(M_{\jmath}, M_{\jmath-1}\right)$. Then the assertion follows from (3.8).

(3.10) Theorem. Let $X$ be as in (3.1). Then (0.3) for $d=n-2$ is bijective.

Proof. The injectivity follows from an argument similar to the proof of (3.1). Here the assumption (3.1.1) is satisfied by (3.8), but (3.1.2) is not, unless the Hodge conjecture is assumed. But (3.1.2) is used only to construct a cycle $\zeta^{\prime} \in \mathrm{CH}_{d+1}\left(X^{\prime}\right)_{\boldsymbol{Q}}$ such that $\eta^{\prime}=\mathrm{Cl}^{\mathrm{NH}}\left(\zeta^{\prime}\right)$. For the proof of this, it is enough to show that the restriction of $\eta^{\prime}$ to an open dense subvariety of $X^{\prime}$ is zero by (1.1.7). Here we may replace $\eta^{\prime}, X^{\prime}$ by $\eta, X$ by definition of $\eta^{\prime}$. Then the

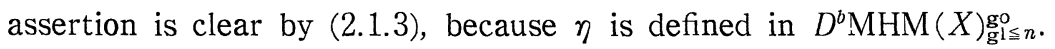

The surjectivity follows from the surjectivity of (3.9.1), because the restriction of $\xi \in \operatorname{Ext}_{X}^{2 d}, g_{1 \leq d i m} X\left(\boldsymbol{Q}_{X}^{H}, \boldsymbol{D}_{X}^{H}(-d)\right)$ to an open dense subvariety is zero by (2.1.3).

Remark. We have a morphism

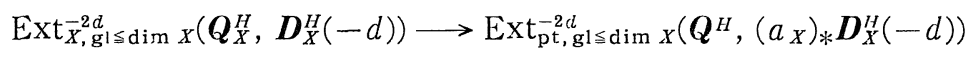

by direct image (2.3) and the restriction morphism (2.8). But it is not clear whether it is bijective. If it is injective, Bloch's conjecture [4] would follow by an argument similar to [17, II, (4.12)].

(3.11) Remark. By (0.8), the injectivity of (0.5) is reduced to the surjectivity of (0.9) and (0.10). The surjectivity of (0.9) would be true if the cycle map of Bloch's higher Chow group $\mathrm{CH}^{p}(X, 1)_{\boldsymbol{Q}}[5]$ to $\operatorname{Ext}_{X, \operatorname{go}}^{2 p-1}\left(\boldsymbol{Q}_{X}^{I}, \boldsymbol{Q}_{X}^{H}(p)\right)$ is surjective, cf. (3.12) below. In general, $\mathrm{CH}^{p}(X, r)_{Q}$ is related with

$$
H_{\mathrm{M} \uparrow}^{i}(X, \boldsymbol{Q}(j))=\operatorname{Ext}_{X, g_{0}}^{2}\left(\boldsymbol{Q}_{X}^{H}, \boldsymbol{Q}_{X}^{H}(j)\right) \quad \text { for } i=2 p-r, j=p
$$

by the cycle map. In fact, with the notation of [loc. cit.], we can construct a cycle map of $\bigcap_{i} \operatorname{Ker} \partial_{i}$ to (3.11.1), and the well-definedness is reduced to its invariance by a deformation parametrized by $\boldsymbol{A}^{1}$, which can be checked using the direct image by a smooth morphism with fiber $\boldsymbol{A}^{1}$. Since an element of $\mathrm{CH}^{p}(X, r)_{Q}$ is represented by a cycle of dimension $\operatorname{dim} X+r-p$, there is no reason to expect the surjectivity of (0.12) for $M=Q_{X}^{I I}, N=Q_{X}^{H}(l), i=2 p-r, n=$ $\operatorname{dim} X$, when $r>p$ (i.e. $i<j$ ). 
For an irreducible variety $X$, let

$$
H_{\mathrm{MH}}^{i}(\operatorname{Rat}(X), \mathbb{Q}(j))=\underset{\lim _{U}}{\longrightarrow} H_{\mathrm{MIi}}^{i}(U, \mathbb{Q}(j)),
$$

where $U$ runs over (smooth) nonempty open subvarieties of $X$, cf. [6]. If we have the surjectivity of the cycle map onto $H_{\mathrm{MH}}^{2}(U, \mathbb{Q}(j))$, then

$$
H_{\mathrm{MH}}^{2}(\operatorname{Rat}(X), \mathbb{Q}(j))=0 \quad \text { for } i>j,
$$

and $H_{\mathrm{MH}}^{2}(\operatorname{Rat}(X), Q(i))$ would be expressed in terms of Tate variations of mixed Hodge structures up to finite covering, and would be related with Milnor $K$ theory. Note that (3.11.3) for $i=2 p, j=p$ is equivalent to the Hodge conjecture by induction on $\operatorname{dim} X$.

(3.12) Remark. We have the surjectivity of (0.9) if

$$
H_{\mathrm{NH}}^{2 p-1}(\operatorname{Rat}(X), \mathbb{Q}(p))=0 \quad \text { for } p>1
$$

for any irreducible variety $X$ (cf. (3.11.2)) using induction on $\operatorname{dim} X$. In fact, we can decrease $\operatorname{dim} X$ and $p$ inductively using the long exact sequence as in (2.14), and reduce to the case $p=1$. So we consider this case in the following.

Let $X$ be a smooth variety. We have

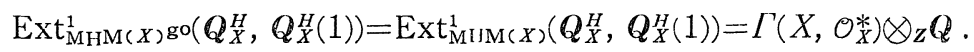

Here the last isomorphism with $Z$-coefficients seems to have been known to some specialists, where the extension in $\operatorname{MHM}(X)$ is replaced by that in the category of admissible variations of mixed Hodge structures. If $X=\mathrm{pt}$, the last isomorphism is due to Carlson, Morgan. This induces a morphism

$$
\alpha: \operatorname{Ext}_{M H M(X)}^{1}\left(Q_{X}^{H}, Q_{X}^{H}(1)\right) \longrightarrow \Gamma\left(X, \mathcal{O}_{X}^{*}\right) \otimes_{Z} Q \mathbb{Q} .
$$

In fact, we have $\alpha: \operatorname{Ext}_{M H M(X)}^{1}\left(Q_{X}^{H}, Q_{X}^{H}(1)\right) \rightarrow \Gamma\left(X^{\text {an }}, \mathcal{O}_{X}^{*}\right.$ an $/ R_{X}$ an $)=\Gamma\left(X^{\text {an }}, \mathcal{O}_{X}^{*}\right.$ an $\left.\otimes Z \mathbb{Q}\right)$, where $R$ denotes the roots of unity of $C$, and $R_{X^{\text {an }}}$ the constant sheaf on $X^{\text {an }}$. Since each $g \in \Gamma\left(X^{\mathrm{an}}, \mathcal{O}_{X^{\mathrm{an}}}^{*} / R_{X^{\mathrm{an}}}\right)$ determines a morphism of $\pi_{1}(X, x)$ into $R$, whose image is a finite group, we get $\Gamma\left(X^{\text {an }}, \mathcal{O}_{X}^{*}\right.$ an $/ R_{X}$ an $)=\Gamma\left(X^{\text {an }}, \mathcal{O}_{X}^{*}\right.$ an $) \otimes_{Z} Q$. Let $e \in \operatorname{Ext}_{\mathrm{MHM}(X)}^{1}\left(Q_{X}^{H}, Q_{X}^{H}(1)\right)$. We have to show $\alpha(e) \in \Gamma\left(X, \mathcal{O}_{X}^{*}\right) \otimes_{Z} Q$. Replacing $X$ by a finite covering, we may assume $\alpha(e) \in \Gamma\left(X^{\mathrm{an}}, \mathcal{O}_{\mathrm{X}^{\text {an }}}^{*}\right)$ by the above argument. By GAGA, it is enough to show that $\alpha(e)$ is meromorphic on a smooth compactification $X^{\prime}$ of $X$. By Remmert-Stein (applied to the graph), we may assume $X^{\prime} \backslash X$ is smooth deleting the singular points. Then the assertion is easily checked using the condition of admissible variation of mixed Hodge structure (i.e. extendability of the Hodge filtration). On the other hand, we have

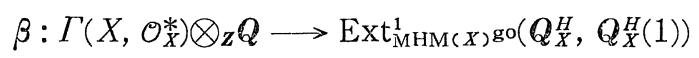

as follows. Let $U=\operatorname{Spec} C\left[t, t^{-1}\right], \quad U^{\prime}=U \backslash\{1\}, \quad Y=X \times U, Y^{\prime}=X \times U^{\prime}$ with 
$j: Y^{\prime} \rightarrow I^{*}$. For $g \subseteq \Gamma\left(X, \mathcal{O}_{X}^{*}\right)$, let $i_{g}: X \rightarrow Y^{*}$ be the inclusion defined by the graph of $g$. If $g \neq 1$, we have a natural morphism $j_{!} Q_{Y^{\prime}}^{H}(1) \rightarrow\left(i_{g}\right)_{*} Q_{X}^{H}(1)$. Taking the

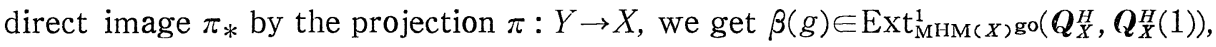
where $\beta(1)$ is defined by 0 . Restricting to each point of $X \backslash g^{-1}(1)$, we can check $\alpha \circ \beta=\mathrm{id}$ (choosing the sign appropriately). In fact, the assertion is reduced to $\int_{1}^{x} d x / x=\log x$ by calculating the period of the mixed Hodge structure corresponding to the extension class. So we get (3.12.2), because $\alpha$ is injective by $[17, \mathrm{I},(4.5)]$.

Let $X$ be an algebraic variety of pure dimension $n, X_{i}(1 \leqq i \leqq r)$ the irreducible components of $X$, and $\operatorname{Rat}(X)^{*}=\mathrm{II}_{i} \operatorname{Rat}\left(X_{i}\right)^{*}$, where $\operatorname{Rat}\left(X_{i}\right)^{*}$ is the group of non zero rational functions on $X_{i}$. Then

$$
\begin{aligned}
\operatorname{Ext}_{\mathrm{MHM}(X) \operatorname{go}}^{1-2 n}\left(\boldsymbol{Q}_{X}^{H}, \boldsymbol{D}_{X}^{H}(1-n)\right) & =\operatorname{Ext}_{\mathrm{MHM}(X)}^{1-2 n}\left(\boldsymbol{Q}_{X}^{H}, \boldsymbol{D}_{X}^{H}(1-n)\right) \\
& =\left\{g \in \operatorname{Rat}(X) * \otimes_{\boldsymbol{Z}} \boldsymbol{Q} \mid \operatorname{div} g=0\right\} .
\end{aligned}
$$

This follows from (3.12.2) and the exact sequence

$$
\begin{aligned}
\operatorname{Ext}_{\mathrm{MHM}(X) \operatorname{go}}^{1-2 n}\left(\boldsymbol{Q}_{X}^{H}, \boldsymbol{D}_{X}^{H}(1-n)\right) & \longrightarrow \operatorname{Ext}_{\mathrm{MHM}(U)}^{1}\left(\boldsymbol{Q}_{U}^{H}, \boldsymbol{Q}_{U}^{H}(1)\right) \\
& \stackrel{\partial}{\longrightarrow} \operatorname{Ext}_{\mathrm{MHM}(Z) \operatorname{go}}^{2-2 n}\left(\boldsymbol{Q}_{Z}^{H}, \boldsymbol{D}_{Z}^{H}(1-n)\right),
\end{aligned}
$$

where $Z=\operatorname{Sing} X$ and $U=X \backslash Z$. In fact, (3.12.5) is reduced to (3.12.2) if $X$ is smooth (i.e. $X=U$ ), and it is enough to show that $\partial$ coincides with $\operatorname{div}: \Gamma\left(U, \mathcal{O}_{U}^{*}\right) \otimes_{Z} \boldsymbol{Q} \rightarrow \oplus \boldsymbol{Q}$, where $\operatorname{Ext}_{\mathrm{MHM}(Z) \operatorname{go}}^{2-2 n}\left(\boldsymbol{Q}_{Z}^{H}, \boldsymbol{D}_{Z}^{H}(1-n)\right)$ is identified with a direct sum of $\boldsymbol{Q}$ by (1.1.7). Since the assertion is local in the classical topology, it is reduced to the case $X=A^{1}, Z=\{0\}$. In this case, $\partial$ is identified with $\left.\operatorname{Ext}_{\mathrm{MHM}(U)}^{1}\left(\boldsymbol{Q}_{U}^{H}, \boldsymbol{Q}_{U}^{H}(1)\right) \rightarrow \operatorname{Hom}_{\mathrm{M}: \mathrm{IS}(Q)}\left(Q^{I I}, H^{1}(U, Q) 、 1\right)\right)$ in $[18,(3.6)]$, and the assertion follows from the proof of [loc. cit. $]$, because it is enough to consider the case $g=\mathrm{id}$.

If (3.12.1) is true for any $X, \operatorname{Ext}_{\mathrm{MHM}(X) \operatorname{go}}^{1-2 d}\left(\boldsymbol{Q}_{X}^{H}, \boldsymbol{D}_{X}^{H}(1-d)\right)$ is generated by $g \in \operatorname{Rat}(Z)^{*} \otimes_{z} \boldsymbol{Q}$ such that $\operatorname{div} g=0$, where $Z$ is a closed subvariety of pure dimension $d$. This suggests a strong relation with higher $K$-theory and Bloch's higher Chow groups [5].

(3.13) Remark. Let $k$ be a field of characteristic zero, and $\mathscr{V}(k)$ the category of reduced and separated varieties over $k$. Assume $k$ is embeddable into $C$, and for any $X \in \mathcal{V}(k)$, there exists an abelian category $\mathscr{M}(X)^{\prime}$ with a forgetful functor rat: $\mathscr{M}(X)^{\prime} \rightarrow \operatorname{Perv}\left(X_{C}, Q\right)$ satisfying the formalism of mixed sheaves (e.g., existence and strictness of weight filtration, semisimplicity of pure objects, stability of its derived category by standard functors compatible with rat, and existence of $\boldsymbol{Q}^{H} \in \mathscr{M}(\operatorname{Spec} k)^{\prime}$ such that $\operatorname{rat}\left(\boldsymbol{Q}^{M}\right)=\boldsymbol{Q}$ and $\boldsymbol{D} \boldsymbol{Q}^{H}$ $=\boldsymbol{Q}^{M}$, etc.), where $\operatorname{Perv}\left(X_{C}, \boldsymbol{Q}\right)$ is the category of $\boldsymbol{Q}$-perverse sheaves on $X_{C}=$ $X \otimes_{k} C=X \times_{\operatorname{spec} k} \operatorname{Spec} C$ with stratification defined over $k$. Here an embedding 
of $k$ into $C$ is chosen. We define the full subcategory $\mathscr{M}(X)$ of $\mathscr{M}(X)^{\prime}$ consisting of the objects of geometric origin as in $[17, \mathrm{II}]$. Then most of the arguments in this paper and [17, II] would apply to this setting. Here the Hodge conjecture would be replaced by the surjectivity of the cycle map

$$
\left.\operatorname{Gr}_{L}^{0} \mathrm{Cl}^{M}: \operatorname{Gr}_{L}^{0} \mathrm{CH}^{p}(X)_{Q} \longrightarrow \operatorname{Hom}_{\operatorname{Mi}(\operatorname{Spec} k}\right)^{\prime}\left(Q^{M}, H^{2 p}\left(a_{X}\right)_{*}\left(a_{X}\right)^{*} Q^{M}(p)\right)
$$

for $X$ smooth projective. If $k$ is a number field, $\mathscr{M}(X)^{\prime}$ might be defined by the category consisting of objects $\left((M, F), K_{\sigma}, K_{l} ; W\right)$ where $(M, F)$ is a filtered holonomic $\mathscr{D}$-Module on $X, K_{\sigma}$ is a $\boldsymbol{Q}$-perverse sheaf on $X_{\sigma}=X^{*} \otimes_{k, \sigma} C$ for each embedding $\sigma: k \rightarrow C, K_{l}$ is an étale $\boldsymbol{Q}_{l}$-perverse sheaf [3] on $\bar{X}=X \bigotimes_{k} \bar{k}$ with action of $\operatorname{Gal}(\bar{k} / k)$ for each prime number $l$, and $W$ is a finite filtration on $M$, $K_{\sigma}, K_{l}$, such that they have comparison isomorphisms as in [9][10][13] (using [3]) and $\left(\left(M \otimes_{k, \sigma} C, F\right), K_{\sigma} ; W\right)$ is a mixed Hodge Module on $X_{\sigma}$ for any $\sigma$. In this case, the surjectivity of (3.13.1) would be equivalent to a conjecture that an absolutely Hodge cycle [9] is algebraic [13].

\section{References}

[1] Beilinson, A., Height pairing between algebraic cycles, Lect. Notes in Math., Springer, Berlin, 1289, (1987), 1-26.

[2] - On the derived category of perverse sheaves, ibid., 27-41.

[3] Beilinson, A., Bernstein, J. and Deligne, P., Faisceaux pervers, Astérisque, 100, (1982).

[4] Bloch, S., Lectures on algebraic cycles, Duke University Mathematical series 4, Durham, 1980.

[5] Algebraic cycles and higher K-theory, Advances in Math., 61 (1986), 267-304.

[6] Bloch, S. and Ogus, A., Gersten's conjecture and the homology of schemes, Ann. Sci. École Norm. Sup. (4), 7 (1974), 181-201.

[7] Deligne, P., Théorie de Hodge I, Actes Congrès Intern. Math. (1970), 425-430; II, Publ. Math. IHES, 40 (1971), 5-58; III, ibid., 44 (1974), 5-77.

[8] Le formalisme des cycles évanescents, in SGA7 XIII and XIV, Lect. Notes in Math., Springer, Berlin, 340, (1973), 82-115, 116-164.

[9] - Valeurs de fonctions $L$ et périodes d'intégrales, in Proc. Symp. in pure Math., 33 (1979), part 2, 313-346.

[10] - Le groupe fondamental de la droite projective moins trois points, in Galois groups over $\boldsymbol{Q}$, Springer, New York, (1989), 79-297.

[11] Grothendieck, A., Hodge's general conjecture is false for trivial reasons, Topology, 8 (1969), 299-303.

[12] Jannsen, U., Deligne homology, Hodge D-conjecture, and motives, in Beilinson's conjecture on special value of L-function, Academic Press, Boston, 1988, 305372.

[13] - Mixed motives and algebraic K-theory, Lect. Notes in Math., Springer, Berlin, 1400 (1990).

[14] Murre, J.P., On the motive of an algebraic surface, J. reine und angew. Math., 409 (1990), 190-204.

[15] Saito, M., Modules de Hodge polarisables, Publ. RIMS, Kyoto Univ., 24 (1988), 
849-995.

[16] Saito, M., Mixed Hodge Modules, Publ. RIMS, Kyoto Univ., 26 (1990), 221-333.

[17] — Hodge conjecture and mixed motives I, preprint MlT, Sept. 1989; II, in Lect. Notes in Math., Springer, Berlin, 1479 (1991), 196-215.

[18] —, Extension of mixed Hodge Modules, Compos. Math., 74 (1990), 209-234.

[19] - Introduction to mixed Hodge Modules, Astérisque, 179-180 (1989), 145162.

[20] - On the formalism of mixed sheaves, preprint RIMS-784, Kyoto Univ., 1991. 
